\title{
Fiskalpolitik als antizyklisches Instrument? Eine Betrachtung der Schweiz
}

\author{
Christoph A. Schaltegger* \\ Universität St. Gallen, Universität Luzern, CREMA und economiesuisse
}

\author{
Martin Weder \\ Universität Luzern, economiesuisse
}

\section{Einleitung}

Folgt man Richard A. Musgrave (1959), bilden die Aufgaben des Staats einen Dreiklang: Allokation, Distribution und Stabilisierung. Die Frage der makroökonomischen Stabilisierung hat dabei in den letzten Monaten stark an Bedeutung gewonnen. Ausgelöst durch die globale Finanzkrise erlebte die Weltwirtschaft einen außergewöhnlich raschen und starken Einbruch. Fast alle Industrienationen befanden sich gleichzeitig in einer Rezession. Gemäß provisorischen Zahlen ist die Weltwirtschaft 2009 zum ersten Mal in der Nachkriegszeit um 1,1 Prozent geschrumpft. In den Industrieländern betrug der Rückgang sogar 3,9 Prozent. Für 2010 wird wieder ein Wachstum der Weltwirtschaft von 3,1 Prozent erwartet (IWF 2010). Dieser starke Einbruch hat sich deutlich auf den Arbeitsmarkt niedergeschlagen. So verdoppelte sich in den USA die Arbeitslosenquote seit Beginn der Rezession im Dezember 2007 von 4,7 auf 10,2 Prozent - und liegt damit auf dem höchsten Stand seit 1983. Die OECD rechnet für 2010 mit einer durchschnittlichen Arbeitslosenquote von 9,0 Prozent in ihren 30 Mitgliedstaaten. Im Jahr 2007 lag diese noch bei 5,6 Prozent. Die ILO hält fest, dass seit Oktober 2008 weltweit mindestens 20 Millionen Arbeitsplätze abgebaut wurden (ILO 2009).

Um die durch den Einbruch der Weltwirtschaft entstehende Nachfragelücke $\mathrm{zu}$ schließen, haben viele Industrienationen umfassende Konjunktur- und Rettungspakete zur Stabilisierung der Wirtschaft beschlossen (für einen Überblick über die nationalen Konjunkturpakete siehe OECD 2009). In den letzten zwei Jahrzehnten hat sich zwar die Ansicht durchgesetzt, dass zur Glättung von Konjunkturschwankungen die Geldpolitik das geeignete Instrument darstellt (CESifo 2009, IWF 2008, Elmendorf und Furman 2008). Als Grund wird angeführt, dass entsprechende fiskalpolitische Maßnahmen oft mit Wahrnehmungs-, Entscheidungs- und Wirkungsverzögerungen konfrontiert sind. Kaum bestritten ist hingegen, dass die Geldpolitik dann die Unterstützung der Fiskalpolitik braucht, wenn diese sich als ineffektiv erwiesen hat oder ihre Mittel vollständig ausgeschöpft sind (IWF 2008). Diese Bedingung scheint in der aktuellen Krise zuzutreffen. Die Notenbanken haben weltweit ihren Leitzins auf ein zum Teil

\footnotetext{
*Korrespondenzadresse: Christoph A. Schaltegger, economiesuisse, Hegibachstrasse 47, CH-8032 Zürich, E-Mail: Christoph.Schaltegger@economiesuisse.ch. - Die Autoren danken einem anonymen Gutachter für wertvolle Hinweise und konstruktive Kritik. Verbleibende Fehler sind alleine den Autoren zuzuschreiben.
} 


\section{Fiskalpolitik als antizyklisches Instrument?}

historisch tiefes Niveau nahe null gesenkt und durch konzertierte Aktionen wiederholt der Volkswirtschaft große Geldmengen zur Verfügung gestellt. Um die Geldmenge zusätzlich noch auszuweiten, wurden unter dem Begriff der quantitativen Lockerung Staatsobligationen und Unternehmensanleihen gekauft.

Grundsätzlich kann die Fiskalpolitik über zwei Arten stabilisierend auf den Konjunkturzyklus wirken: erstens über automatische Stabilisatoren und zweitens über eine diskretionäre Fiskalpolitik. Die Wirkungsweise der automatischen Stabilisatoren hängt dabei vom Transfersystem wie etwa der Arbeitslosenversicherung, der Progressivität des Steuersystems und weiteren institutionellen Regeln, wie beispielsweise einer Schuldenbremse ab (Feld und Kirchgässner 2008). Die diskretionäre Fiskalpolitik bedarf dagegen expliziter politischer Entscheidungen im Bereich der Ausgaben- und/oder der Steuerpolitik. Diskretionäre Konjunkturstimuli schneiden in bisherigen Studien anhand der drei oft verwendeten Kriterien „zielgenau, zeitgerecht und zeitlich beschränkt“ schlechter ab als die automatischen Stabilisatoren (IWF 2008, Elmendorf und Furman 2008, Stone und Cox 2008).

Im Folgenden wird die Stabilisierungswirkung der Fiskalpolitik der Schweiz über die letzten 50 bzw. 30 Jahre analysiert. Dazu wird zunächst die Fiskalpolitik des Bundes und danach die der Kantone untersucht. Es zeigt sich, dass in etwa 60\% der Jahre der Bundeshaushalt tatsächlich antizyklisch wirkte. In Rezessionsjahren wirkte die Fiskalpolitik des Bundes immer antizyklisch. In den Kantonen ist die Quote etwas tiefer. In einem zweiten Schritt unterscheiden wir zwischen der Wirkung der automatischen Stabilisatoren und der diskretionären Fiskalpolitik als antizyklisches Instrument. Dabei wird die quantitativ bedeutendere Rolle der automatischen Stabilisatoren im Vergleich zur diskretionären Fiskalpolitik deutlich. Drittens betrachten wir die makroökonomischen Effekte der antizyklischen Fiskalpolitik. Es zeigt sich, dass die automatischen Stabilisatoren als effektive Konjunkturstütze wirken, während die Impulse der diskretionären Fiskalpolitik einen geringeren Einfluss auf den Konjunkturverlauf ausüben. Abschließende Bemerkungen über die Erfahrungen der antizyklischen Fiskalpolitik in der Schweiz runden den Beitrag ab.

\section{Die Stabilisierungswirkung der Schweizer Fiskalpolitik}

Im Zentrum der politischen Debatte stehen die so genannten Multiplikatoreffekte fiskalpolitischer Maßnahmen. Welches sind die ausgelösten Veränderungen des Bruttoinlandsprodukts (BIP) in Beziehung zum Umfang der getroffenen Maßnahmen? Die empirische Literatur zu den internationalen Erfahrungen mit der Stabilisierungswirkung der Fiskalpolitik ist gleichermaßen umfangreich wie uneinheitlich. Dies liegt unter anderem an den unterschiedlichen methodischen Verfahren. Der IWF (2008) unterscheidet zwischen drei verschiedenen Ansätzen: einem „narrativen Ansatz“, der Politikänderungen aus der historischen Erfahrung des Gesetzgebungsprozesses identifiziert (Ramey und Shapiro 1998), einem „Fallstudienansatz“, der die Wirkung eines „natürlichen Experiments“, beispielsweise eines Steuerrabatts, untersucht (Shapiro und Slemrod 2002 für die Wirkung des 2001 Steuerrabatts in den USA) und einem Ansatz, über strukturelle Vektorautoregressionen den Einfluss fiskalpolitischer Schocks auf die BIP-Fluktuationen zu untersuchen. Die erwähnte Studie des IWF (2008) untersucht in einer Zusammenstellung 41 Länder über den Zeitraum von 1970 bis 2007 


\section{Christoph A. Schaltegger und Martin Weder}

und stellt fest, dass Impulsprogramme zwar einen positiven Einfluss auf die Wirtschaft haben. Dieser Effekt ist aber bei einem Impuls von einem Prozent des BIP mit einer durchschnittlichen Erhöhung des BIP von 0,1 Prozent im Jahr der Krise gering. Auch der Gesamteffekt über die nächsten drei Jahre ist mit einer Erhöhung des BIP um insgesamt 0,5 Prozent relativ klein. Die Studie zeigt außerdem, dass Fiskalimpulse umso eher versickern, je größer die Sparquote der Haushalte und je offener die Volkswirtschaft (Abfluss über Importe) ist. Die beschlossenen Maßnahmen finden zweitens oft eine permanente statt temporäre Anwendung und erhöhen somit die Schulden wesentlich. Auch Reinhart und Rogoff (2009) stellen fest, dass in den Industrienationen seit dem Zweiten Weltkrieg die Verschuldung im Zuge einer Finanzkrise innerhalb von drei Jahren um durchschnittlich 86 Prozent zugenommen hat. Andere Untersuchungen (OECD 2009) weisen insbesondere bei offenen Volkswirtschaften für zusätzliche Staatsausgaben einen tiefen Multiplikatoreffekt zwischen 0,3 und 0,8 aus. Bezogen auf die Schweiz würde das bedeuten, dass für jeden zusätzlichen Franken, den der Staat ausgibt, die Wirtschaft nur um 0,3 bis 0,8 Franken wächst. Barro (1974, 2009) kritisiert die Annahme hoher Multiplikatoreffekte, weil Haushalte und Unternehmen die Staatsdefizite von heute als Steuererhöhungen von morgen interpretieren. Dementsprechend werden sie bereits heute ihre Ersparnisse im gleichen Ausmaß erhöhen, wie der Staat zusätzliche Schulden macht. Es resultiert ein Nullsummenspiel: Der Impuls des Staates wird von den privaten Akteuren vollständig absorbiert. Studien, welche mit Vektorautoregressionen arbeiten, gibt es vor allem für OECD- und EU-Staaten (z.B. Perotti 2005 für OECD-Staaten, Cwik und Wieland 2009 für 11 EU-Länder). Insbesondere zu den USA finden sich zahlreiche Publikationen (z.B. Blanchard und Perotti 2002).

Für die Schweiz liegen Simulationsrechnungen für das Makromodell der KOFETH vor (Frick, Graff und Hartwig 2009). Diese gehen von einem Multiplikatoreffekt von 1,7 aus, sind aber mit den oben erwähnten empirischen Arbeiten nicht vergleichbar. Balastèr (2009) verwendet einen narrativen Ansatz, um die Impulsprogramme des Bundes und deren Wirkung seit 1970 darzustellen. Jordan (1994) untersucht die Wirkung der Bundesfinanzpolitik der 1980er und 1990er Jahre. Er kommt zum Ergebnis, dass der Bund eine stark prozyklische Fiskalpolitik betrieben habe. Analysen, welche mit Hilfe von Vektorautoregressionen den Einfluss fiskalpolitischer Schocks auf das Wirtschaftswachstum untersuchen, existieren hingegen bisher nicht.

Die rechtlichen Voraussetzungen für eine Stabilisierungspolitik in der Schweiz haben sich allmählich entwickelt (Frey 2007). Erste Ansätze einer aktiven Konjunkturpolitik des Bundes stammen aus dem Jahr 1942 unter dem Eindruck der Weltwirtschaftskrise der 1930er Jahre. Der eigentliche Konjunkturartikel wurde aber erst 1978 von Volk und Ständen im zweiten Anlauf angenommen. In der Schweizer Bundesverfassung wird heute in Art. 100 festgehalten, dass der Bund Maßnahmen für eine ausgeglichene konjunkturelle Entwicklung zu treffen hat, insbesondere zur Verhütung und Bekämpfung von Arbeitslosigkeit und Teuerung (Absatz 1). Er berücksichtigt dabei die wirtschaftliche Entwicklung der einzelnen Landesgegenden und arbeitet mit den Kantonen und der Wirtschaft zusammen (Absatz 2).

Wie wurden diese verfassungsrechtlichen Vorgaben umgesetzt? Welche fiskalpolitischen Impulse wurden über die letzten 50 Jahre vom Bundes- und den Kantonshaushalten auf die Konjunktur abgegeben? 


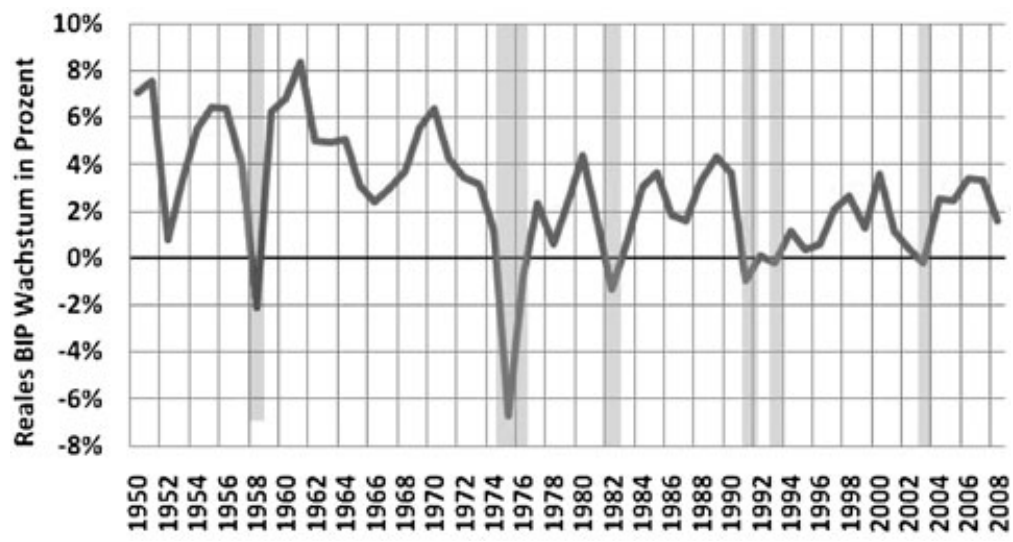

Abbildung 1 Entwicklung des realen Wirtschaftswachstums in der Schweiz (1950-2008) Quelle: Seco (2009)

\subsection{Analyse der Reaktion der Fiskalpolitik während Rezessionen}

Zur Analyse der Schweizer Fiskalpolitik während Rezessionen, müssen zuerst die Rezessionsphasen identifiziert werden. Nach einer gängigen, aber nicht unumstrittenen Definition liegt eine Rezession vor, wenn das BIP in zwei aufeinander folgenden Quartalen einen Rückgang verzeichnet. Da im Folgenden Jahreswerte verwendet werden, haben wir rezessive Phasen als Jahre mit einem negativen realen BIP-Wachstum definiert. ${ }^{1}$ Gemäß dieser Definition durchlief die Schweiz seit 1950 sechs Rezessionsphasen: 1958, 1975-1976, 1982, 1991, 1993, 2003 (vgl. Abbildung 1).

Kantonsdaten stehen für den Zeitraum 1971 bis 2005 zur Verfügung. Eine Rezession wurde analog als Rückgang des realen kantonalen Volkseinkommens ${ }^{2}$ innerhalb eines Jahres definiert. Gemäß dieser Definition haben über den gesamten Zeitraum betrachtet alle Kantone mehrere Rezessionen erlebt, wobei es deutliche Unterschiede gibt. So war der Kanton Neuenburg beispielsweise in den 70er-Jahren stark von der Krise der Uhrenindustrie betroffen, während der Kanton Solothurn in den 90er-Jahren aufgrund der hohen Bedeutung des industriellen Sektors mehrere Jahre mit schrumpfendem Volkseinkommen verzeichnete. Außerdem haben kleinere Kantone gelegentlich mit strukturellen Problemen zu kämpfen, inklusive eines Rückgangs der ständigen Wohnbevölkerung. Ein Rückgang des kantonalen Volkseinkommens wurde in diesen 35 Jahren in sechs (Nidwalden, St. Gallen, Waadt) bis 15 Perioden (Neuenburg) registriert. Betrachtet man nur die Rezessionsjahre, so ergeben sich insgesamt 243 Beobachtungen. (vgl. Tabelle 1).

1. Quartalsdaten sind erst ab 1965 erhältlich. Die so definierten Rezessionen decken sich aber vollumfänglich mit der Verwendung von Jahresdaten, so dass keine Rezession „übersehen“ wird. Auf umfangreichere, detailliertere Definitionen von Rezessionen, wie sie in den USA beispielsweise das National Bureau of Economic Research (NBER) verwendet, wurde nicht zuletzt aufgrund fehlender Daten verzichtet. Das NBER definiert eine Rezession als „a significant decline in economic activity spread across the country, lasting more than a few months, normally visible in GDP growth, real personal income, employment (non-farm payrolls), industrial production and wholesale retail sales."

2. Kantonale BIP-Daten sind erst ab 1990 erhältlich. 


\section{Christoph A. Schaltegger und Martin Weder}

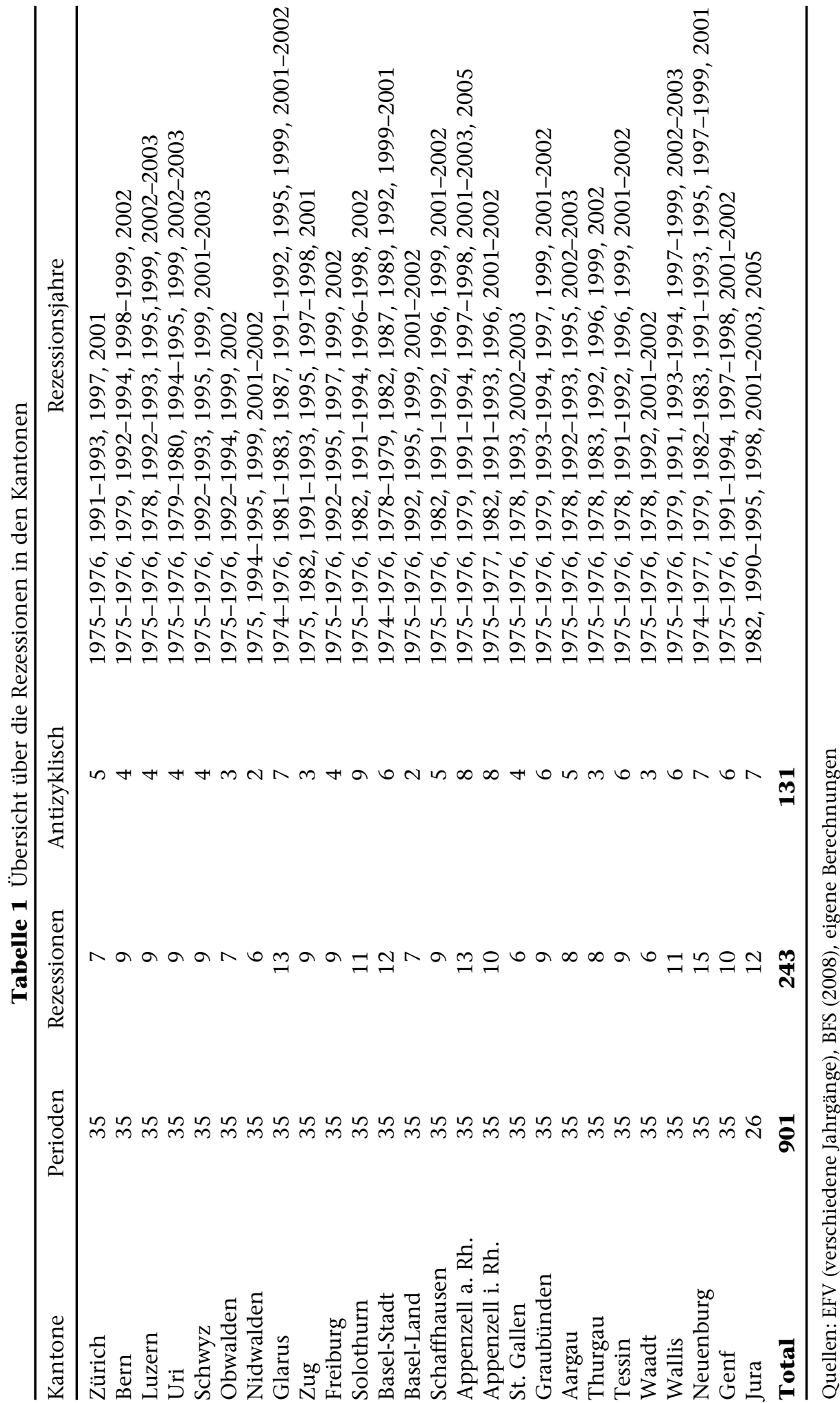




\section{Fiskalpolitik als antizyklisches Instrument?}

Zweitens geht es um die Definition des Fiskalimpulses. In Anlehnung an den IWF (2008) definieren wir den Fiskalimpuls als Veränderung der realen Primärbilanz gegenüber dem Vorjahr für alle Perioden $t=1951-2007$ für den Bund bzw. $t=$ 1971-2005 für die Kantone als

$$
\mathrm{g}_{\mathrm{t}}=\underbrace{\left(\mathrm{r}_{\mathrm{t}}-\mathrm{p}_{\mathrm{t}}\right)-\left(\mathrm{r}_{\mathrm{t}-1}-\mathrm{p}_{\mathrm{t}-1}\right)}_{\text {Primärbilanz }}+\underbrace{\left(\mathrm{e}_{\mathrm{t}}-\mathrm{a}_{\mathrm{t}}\right)-\left(\mathrm{e}_{\mathrm{t}-1}-\mathrm{a}_{\mathrm{t}-1}\right)}_{\text {Sozialversicherungsbilanz }}
$$

wobei $r_{t}$ für die realen Staatseinnahmen im Verhältnis zum realen BIP in Periode $\mathrm{t}$ und $\mathrm{p}_{\mathrm{t}}$ für die Primärausgaben (Gesamtausgaben abzüglich Passivzinsen) im Verhältnis zum BIP in Periode $t$ stehen. Der Ausdruck $r_{t}-p_{t}$ ist somit die Primärbilanz in Periode t. Bei der Berechnung des Fiskalimpulses auf Bundesebene wurden zusätzlich noch der Einfluss der vier staatlichen Sozialversicherungen Alters- und Hinterlassenenversicherung (AHV), Invalidenversicherung (IV), Arbeitslosenversicherung (ALV) und Erwerbsersatzordnung (EO) miteinbezogen. Der Term $e_{t}$ steht dabei für die Einnahmen der Sozialversicherungen im Verhältnis zum BIP, $a_{t}$ für die entsprechenden Ausgaben.

Im Unterschied $\mathrm{zu}$ ähnlichen Analysen musste darauf verzichtet werden, konjunkturell bereinigte Werte für die Primärbilanz zu verwenden. Dies liegt unter anderem daran, dass die entsprechenden Daten für den Bund nicht bis 1950 zurückreichen. Für die Kantone existieren weder Daten über das potenzielle noch über das konjunkturell bereinigte BIP bzw. Volkseinkommen. Blanchard (1990) und Jordan (1994) weisen zudem darauf hin, dass die Meinungen zur Verwendung von konjunkturell bereinigten Werten kontrovers sind, weil davon ausgegangen wird, dass das BIP um einen konstanten Wachstumspfad fluktuiert. Zudem gibt es verschiedene Methoden, um das potenzielle und das konjunkturell bereinigte BIP zu berechnen. Jordan (1994) hält fest, dass je nach Berechnungsmethode des konjunkturell bereinigten BIP, hohe aktuelle Defizite auch als unrealistische Überschüsse erscheinen können. Auch die Verwendung von Elastizitäten ist indessen nicht ganz unproblematisch. Mangels Alternativen orientieren wir uns wiederum an der Publikation des IWF, welcher sowohl mit auf Elastizitäten basierten Impulsen als auch mit konjunkturell bereinigten Werten arbeitet (IWF 2008).

Abbildung 2 zeigt die vom Bundeshaushalt und den Sozialversicherungen abgegebenen Impulse im Vergleich zum realen BIP-Wachstum. Der Fiskalimpuls ist dabei definiert als Veränderung der Primärbilanz gegenüber dem Vorjahr. Wenn in einer Rezession die Steuereinnahmen abnehmen und die Ausgaben aufgrund von Konjunkturprogrammen erhöht werden, so verschlechtert dies die Primärbilanz. In Abbildung 2 würde sich in diesem Fall der Balken für den Fiskalimpuls im negativen Bereich befinden; die Finanzpolitik wäre antizyklisch. Mit anderen Worten: Eine antizyklische Fiskalpolitik ist grafisch so illustriert, dass Impuls und Wirtschaftswachstum immer das gleiche Vorzeichen aufweisen und somit in die gleiche Richtung zeigen. Ein negativer Wert für den Fiskalimpuls bei positivem Wirtschaftswachstum müsste entsprechend als prozyklische Politik interpretiert werden. Betrachtet werden jeweils die einzelnen Jahre. ${ }^{3}$ Dadurch ergibt sich, dass

3. Mit dieser Methode wird z.B. der Auslastungsgrad der Wirtschaft nicht berücksichtigt. Solche Indikatoren betreffen in der Regel allerdings nur den sekundären Sektor, dessen Anteil an der Gesamtwertschöpfung in den letzten Jahren kontinuierlich abgenommen hat. Der 


\section{Christoph A. Schaltegger und Martin Weder}

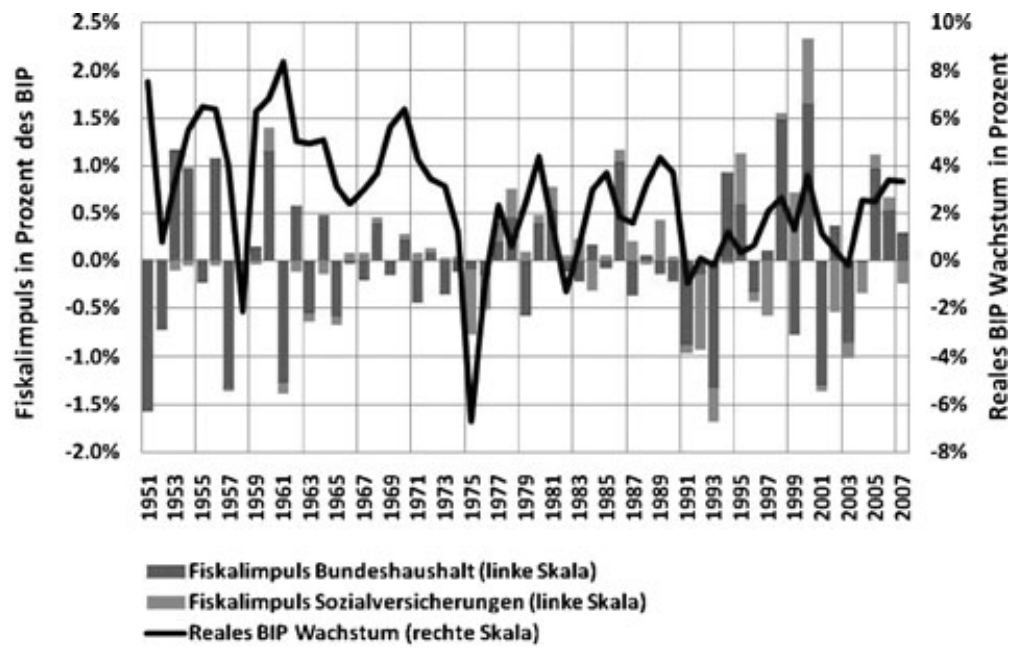

Abbildung 2 Fiskalimpuls des Bundes und der Sozialversicherungen (1951-2007)

Quellen: EFV (verschiedene Jahrgänge), BFS (2008), BSV (2009), Seco (2009), eigene Berechnungen.

die Fiskalpolitik zwischen 1951 und 2007 in etwas mehr als 60 Prozent der Fälle antizyklisch und in knapp 40 Prozent der Fälle prozyklisch war.

In der Vergangenheit wurde der Bund für seine prozyklische Finanzpolitik wiederholt kritisiert. ${ }^{4}$ Unterteilt nach Jahrzehnten lässt sich jedoch seit den 90erJahren eine Entwicklung in Richtung antizyklischer Fiskalpolitik beobachten. Seit der Einführung der Schuldenbremse im Jahr 2003 war die Fiskalpolitik des Bundes sogar durchwegs antizyklisch. Ersichtlich ist auch, dass mit dem Ausbau der Sozialversicherungen der Impuls aus diesem Bereich im Verhältnis zu den über das Budget beschlossenen Maßnahmen laufend größer wurde.

Abbildung 3 zeigt die Fiskalimpulse der Kantone für den Zeitraum 19712005. Der ungewichtete Fiskalimpuls entspricht dabei dem Durchschnittswert der 26 Kantone, während der gewichtete Wert die unterschiedliche Größe der Volkswirtschaften (ausgedrückt durch die Wohnbevölkerung) berücksichtigt. In den meisten Jahren führt dies jedoch kaum zu nennenswerten Unterschieden. Verglichen mit Grafik 2 wird deutlich, dass der Impuls der Kantone tendenziell wesentlich kleiner ist. Während die Impulse beim Bund auch ohne Einbezug der Sozialversicherungen häufig Werte von über 1 Prozent des BIP annehmen, erreicht der Impuls der Kantone nur selten die Schwelle von 0,5 Prozent. Damit bestätigt sich die verfassungsrechtlich bestimmte Führungsrolle des Bundes für die Stabilisierungspolitik, auch wenn die Summe der Ausgaben aller Kantone im Verhältnis zu den Bundesausgaben einen höheren Anteil am BIP ausmacht (zuletzt 15 bzw. 11 Prozent). Analog zur Entwicklung auf Bundesebene hat jedoch auch bei

Dienstleistungssektor macht hingegen über 70\% des BIP aus. Die aktuelle Krise ist in den Daten noch nicht enthalten (1951-2007). Insofern war die Auslastung der Wirtschaft nur in einem Fall $(1975 / 76)$ deutlich unter dem Potenzial.

4. Beispiele dafür sind Frey (2007), Frick und Lampart (2004), Lampart (2005), Jordan (1994) oder auch die OECD (2002). Für einzelne Perioden gibt es jedoch auch Gegenbeispiele, so z.B. Jaeger und Schips (1980) für die 1970er Jahre. 


\section{Fiskalpolitik als antizyklisches Instrument?}

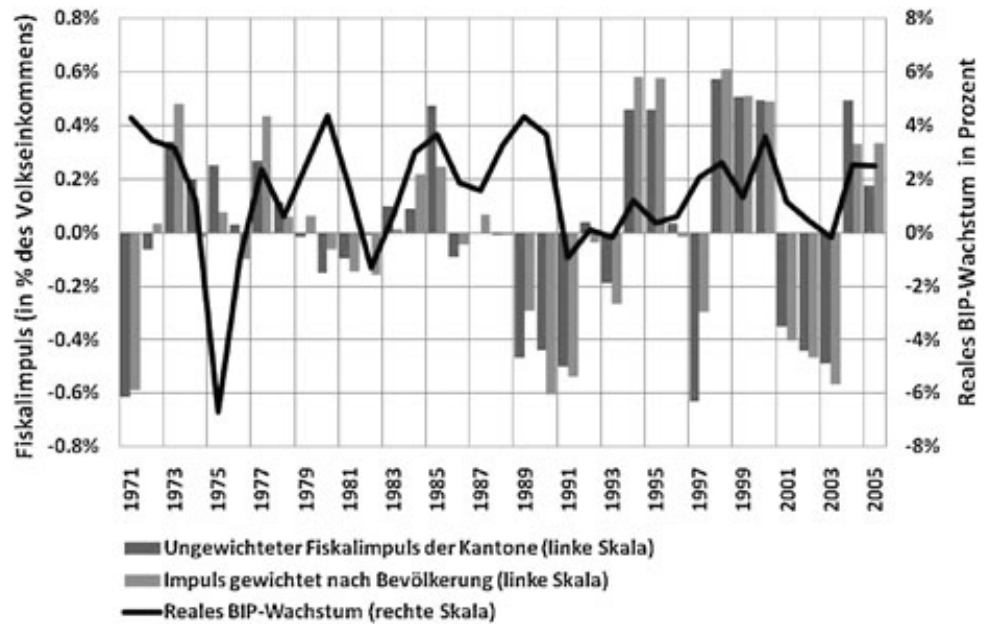

Abbildung 3 Fiskalimpuls der Kantone (1970-2005)

Quellen: EFV (verschiedene Jahrgänge), BFS (2008), eigene Berechnungen

den Kantonen der Impuls im Verhältnis zum Wirtschaftswachstum über die Zeit zugenommen.

Bisherige Untersuchungen kamen zum Schluss, dass die Kantone in der Vergangenheit oft eine prozyklische Finanzpolitik verfolgten. Es wird kritisiert, die verfolgte Fiskalpolitik der Kantone hätte die jeweilige wirtschaftliche Lage noch verschärft. Ammann (2002) hat drei Perioden identifiziert, während derer die Kantone eine klar prozyklische Budgetpolitik verfolgten: 1970-1972, 1988-1990 und 1993-1995. Frick und Lampart (2004) untersuchten die Periode von 1988-2004 und stellten dabei fest, dass sich die Kantone in etwa gleich häufig prozyklisch wie antizyklisch verhielten. Verglichen mit dem Bund waren sie damit etwas häufiger prozyklisch.

\subsection{Unterscheidung zwischen automatischen Stabilisatoren und diskretionärer Fiskalpolitik}

Nachdem wir die Fiskalimpulse identifiziert haben, stellt sich die Frage der Zusammensetzung. Wie oben erwähnt, setzt sich der gesamte Fiskalimpuls aus einer automatischen Komponente über das Steuersystem, die Sozialversicherungen oder die Schuldenbremse und einer diskretionär bestimmten Größe aus der aktiven Konjunkturpolitik zusammen. Der Fiskalimpuls g kann illustrativ wie folgt dargestellt werden (siehe auch IWF 2008):

$$
\begin{aligned}
g_{t} & =\left(r_{t}\left(y_{t}\right)-p_{t}\left(y_{t}\right)\right)-\left(r_{t-1}\left(y_{t-1}\right)-p_{t-1}\left(y_{t-1}\right)\right) \\
& =\underbrace{\left(r_{t}\left(y_{t}\right)-p_{t}\left(y_{t}\right)\right)-\left(r_{t}\left(y_{t-1}\right)-p_{t}\left(y_{t-1}\right)\right)}_{\text {Automatischer Stabilisator }}+\underbrace{\left(r_{t}\left(y_{t-1}\right)-p_{t}\left(y_{t-1}\right)-\left(r_{t-1}\left(y_{t-1}\right)-p_{t-1}\left(y_{t-1}\right)\right)\right.}_{\text {Diskretionärer Fiskalimpuls }}
\end{aligned}
$$

wobei $r_{t}\left(y_{t}\right)-p_{t}\left(y_{t}\right)$ den Primärsaldo in Periode $t$ in Abhängigkeit der Wirtschaftslage y darstellt. Der Ausdruck $r_{t}\left(y_{t-1}\right)-p_{t}\left(y_{t-1}\right)$ ist ein Hilfsterm. Er zeigt 


\section{Christoph A. Schaltegger und Martin Weder}

einen hypothetischen Primärsaldo in Periode $t$, der resultieren würde, wenn sich die Wirtschaft y so entwickelt hätte wie in Periode $t-1$. Der erste Klammerausdruck $\mathrm{r}_{\mathrm{t}}\left(\mathrm{y}_{\mathrm{t}}\right)-\mathrm{p}_{\mathrm{t}}\left(\mathrm{y}_{\mathrm{t}}\right)-\left(\mathrm{r}_{\mathrm{t}}\left(\mathrm{y}_{\mathrm{t}-1}\right)-\mathrm{p}_{\mathrm{t}}\left(\mathrm{y}_{\mathrm{t}-1}\right)\right)$ zeigt damit den konjunkturellen Anteil der Veränderung des Primärsaldos - also des Fiskalimpulses -, während der Ausdruck $\mathrm{r}_{\mathrm{t}}\left(\mathrm{y}_{\mathrm{t}-1}\right)-\mathrm{p}_{\mathrm{t}}\left(\mathrm{y}_{\mathrm{t}-1}\right)-\left(\mathrm{r}_{\mathrm{t}-1}\left(\mathrm{y}_{\mathrm{t}-1}\right)-\mathrm{p}_{\mathrm{t}-1}\left(\mathrm{y}_{\mathrm{t}-1}\right)\right)$ die diskretionären Maßnahmen definiert. Der Hilfsterm $r_{t}\left(y_{t-1}\right)-p_{t}\left(y_{t-1}\right)$ ist weiter definiert als

$$
\mathrm{r}_{\mathrm{t}}\left(\mathrm{y}_{\mathrm{t}-1}\right)-\mathrm{p}_{\mathrm{t}}\left(\mathrm{y}_{\mathrm{t}-1}\right)=\mathrm{r}_{\mathrm{t}-1} *\left(1+\left[\mathrm{y}_{\mathrm{t}-1} * \varepsilon_{\mathrm{r}}\right]\right)-\mathrm{p}_{\mathrm{t}-1}
$$

Zur Berechnung des hypothetischen Primärüberschuss $\mathrm{r}_{\mathrm{t}}\left(\mathrm{y}_{\mathrm{t}-1}\right)-\mathrm{p}_{\mathrm{t}}\left(\mathrm{y}_{\mathrm{t}-1}\right)$ in Periode $t$ werden zunächst die Einnahmen in Prozent des BIP der Vorperiode t-1 multipliziert mit der Wachstumsrate der Wirtschaft des letzten Jahres $\mathrm{y}_{\mathrm{t}-1}$ und der langfristigen Elastizität der Einnahmen $\varepsilon_{\mathrm{r}}{ }^{5}$ Die Elastizität $\varepsilon_{\mathrm{r}}$ wurde über den gesamten Zeitraum von 1951-2007 bzw. 1971-2005 berechnet. Sie liegt bei 1,17 für den Bund und mit der Ausnahme von Uri auch bei allen Kantonen deutlich über eins. Das Ergebnis für den Bund deckt sich mit den Annahmen, die von der Eidgenössischen Finanzverwaltung für das Budget und den Finanzplan gemacht werden. In einem aktuellen Arbeitspapier wird die Elastizität auf 1,19 beziffert (Bruchez, Colombier, Geier, Schlaffer und Rey 2009). ${ }^{6}$ Die Einnahmen reagieren damit relativ elastisch auf die konjunkturelle Entwicklung. Von den hypothetischen Einnahmen werden die Ausgaben der Vorperiode $t-1$ subtrahiert. Damit wird angenommen, dass es in den Bundes- und Kantonshaushalten kaum Ausgabenpositionen gibt, die institutionell an den Konjunkturverlauf gekoppelt sind. Ein ähnliches Vorgehen wählt Jordan (1994). Mit Ausnahme der Ausgaben für die Arbeitslosenversicherung definiert er alle übrigen Veränderungen bei den Ausgaben als rein diskretionär. ${ }^{7}$ Wir haben für die Zurechnung der Sozialversicherungen zum Fiskalimpuls zwei verschiedene Methoden gewählt. Im ersten Fall wurde der Impuls der Sozialversicherungen analog zu oben in einen automatischen und in einen diskretionären Teil zerlegt. Bei der zweiten Methode wurde der gesamte Impuls vollständig als automatischer Stabilisator betrachtet. Die Überlegung dahinter ist, dass ein Ausbau von Sozialversicherungsleistungen in Periode $\mathrm{t}$ ab Periode $\mathrm{t}+1$ als automatischer Stabilisator zu wirken beginnt. Das Ausmaß der automatischen Stabilisatoren wird bei diesem Vorgehen aber eventuell überschätzt.

5. Das Trendwachstum spielt bei dieser Zerlegung in einen diskretionären und einen automatischen Impuls keine Rolle. Wie Gleichung (3) zeigt, bleiben die Einnahmen konstant, wenn die Wirtschaft nicht wächst $\left(\mathrm{y}_{\mathrm{t}-1}=0\right)$. Weil die Ausgaben im Grundmodell als diskretionär betrachtet werden, würde es in dieser Situation weder einen diskretionären noch einen automatischen Impuls geben. Die Fiskalpolitik wäre gegenüber der Vorperiode unverändert. Bei konstantem Trendwachstum hingegen entsteht aufgrund der Elastizität $\left(\varepsilon_{\mathrm{r}}>1\right)$ eine Wachstumsdividende, die vollständig als automatischer Stabilisator dargestellt wird.

6. Bei der Schweizer Schuldenbremse werden ebenfalls hypothetische Einnahmenwerte berechnet, korrigiert über einen so genannten Konjunkturfaktor (K-Faktor). Der K-Faktor ist definiert als das Verhältnis von geschätztem trendmäßigem Bruttoinlandsprodukt zu geschätztem aktuellem Bruttoinlandsprodukt. Das Trend-BIP wird mit Hilfe einer modifizierten Variante des HodrickPrescott-Filters bestimmt (Bruchez 2003). Der Hodrick-Prescott Filter bewirkt jedoch, dass das TrendBIP in Richtung der letzten Beobachtungen verzerrt ist (sog. „Endpunkt“-Problematik, EFV 2004). Wir haben aus diesen Gründen mit langfristigen Elastizitäten und nicht mit BIP-Trendwerten gerechnet.

7. Mit dieser Definition wird der Umfang der diskretionären Maßnahmen möglicherweise über- und derjenige der automatischen Stabilisatoren unterschätzt. 


\section{Fiskalpolitik als antizyklisches Instrument?}

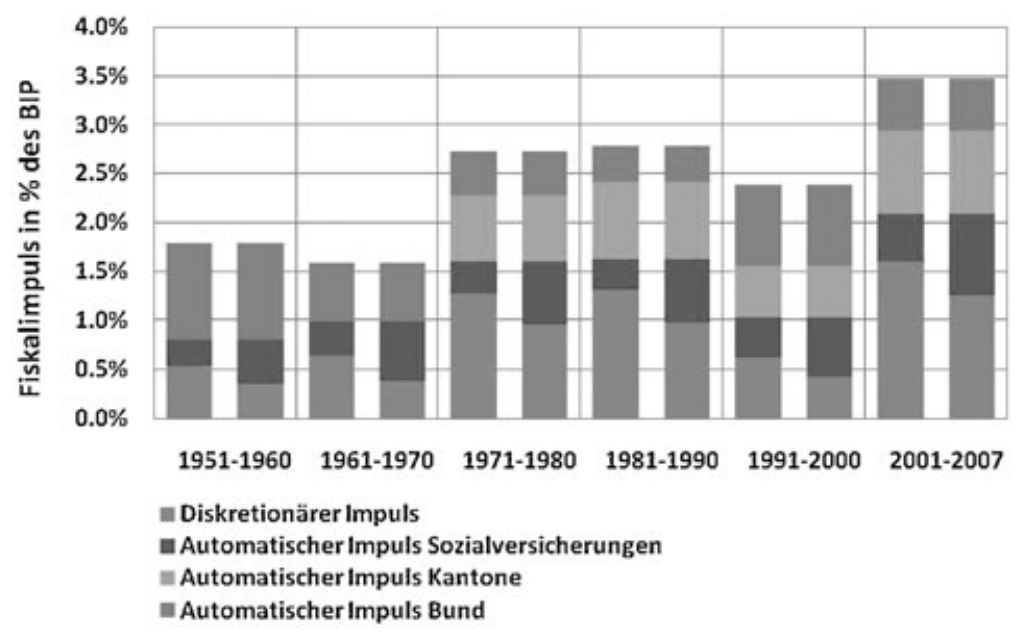

Abbildung 4 Stetiger Ausbau der automatischen Stabilisatoren

Quellen: EFV (verschiedene Jahrgänge), BFS (2008), BSV (2009), Seco (2009), eigene Berechnungen

Abbildung 4 zeigt das Verhältnis von automatischen Stabilisatoren und diskretionären Maßnahmen unterteilt nach Jahrzehnte. Die linke Säule unterteilt den Impuls der Sozialversicherungen analog zum Finanzhaushalt von Bund und Kantonen in zwei Teile, während die rechte Säule die Sozialversicherungen vollständig als automatischen Stabilisator behandelt. Die Grafik zeigt, dass die automatischen Stabilisatoren sowohl auf Bundes- wie auch auf Kantonsebene über die letzten Jahre stark ausgebaut wurden. ${ }^{8}$ Ihr Impuls hat sich seit Anfang der 60er Jahre kontinuierlich von durchschnittlich 0,9 auf 1,9 Prozent des BIP verdoppelt. Dies liegt zum einen in der Ausdehnung der generellen Staatstätigkeit (Erhöhung der Staats- und Fiskalquote). Fatás und Mihov (2001) argumentieren beispielsweise, dass die Höhe der Staatsquote mit dem Ausmaß der automatischen Stabilisatoren auch international korreliert. Zum andern liegt die Stärkung der automatischen Stabilisatoren im Ausbau der Sozialversicherungen und jüngst in der Einführung der Schuldenbremse begründet.

Seit 1951 hat sich gleichzeitig auch der diskretionäre Impuls von 0,6 auf 1,6 Prozent des BIP erhöht. Solche permanenten und diskretionären Fiskalimpulse verursachen eine steigende Staats- und Fiskalquote und stärken damit nachgelagert auch die automatischen Stabilisatoren. Gleichzeitig reduzieren sich mit der Ausdehnung der Staatstätigkeit aber die langfristigen Wachstumsaussichten (IWF 2008).

Über die Zeit lässt sich kein Trend bezüglich der Anteile der automatischen Stabilisatoren und der diskretionären Impulse feststellen. Während die Schwankungen von Jahr zu Jahr zum Teil erheblich sind, liegt der Anteil der automatischen Stabilisatoren unter Einbezug von Bund, Kantonen und Sozialversicherungen je nach Berechnungsmethode zwischen 60 und 72 Prozent. Der Impuls der automatischen Stabilisatoren ist damit durchschnittlich etwa

8. Daten für die Kantone sind erst ab 1970 verfügbar. Die Graphik enthält für die Intervalle 1951-1960 und 1961-1970 somit nur den Impuls des Bundes und der Sozialversicherungen. 


\section{Christoph A. Schaltegger und Martin Weder}

Tabelle 2 Finanzpolitische Maßnahmen des Bundes als Reaktion auf Krisen seit 1950

\begin{tabular}{lccccc}
\hline \multirow{2}{*}{ Maßnahmen } & Zeitpunkt & Ausgaben & $\%$-BIP & Impuls & $\begin{array}{c}\text { Auftrags- } \\
\text { volumen }\end{array}$ \\
\hline Arbeitsbeschaffungsprogramme I - III & Juni 1975 & 2,10 Mrd. & $1,4 \%$ & $1,3 \%$ & $2,0 \%$ \\
Milderungsmaßnahmen & Dez. 1978 & 0,12 Mrd. & $0,1 \%$ & $0,7 \%$ & - \\
Beschaffungsprogramm & März 1983 & 0,90 Mrd. & $0,4 \%$ & $0,0 \%$ & $1,1 \%$ \\
Investitionsbonus 1993 & März 1993 & 0,30 Mrd. & $0,1 \%$ & $1,7 \%$ & $0,4 \%$ \\
Investitionsbonus 1997 & März 1997 & 0,48 Mrd. & $0,1 \%$ & $0,5 \%$ & $0,6 \%$ \\
Stabilisierungsmaßnahmen I - III & Dez. 2008 & 2,09 Mrd. & $0,4 \%$ & $?$ & $?$ \\
\hline
\end{tabular}

Quellen: Balastèr (2009), eigene Berechnungen

doppelt so groß wie jener von kurzfristig beschlossenen Maßnahmen. Dabei muss jedoch weiter differenziert werden. Auf Bundesebene ist der Impuls der automatischen Stabilisatoren mit 0,6 Prozent des BIP doppelt so groß wie der diskretionäre Effekt (0,3 Prozent). Bei den Sozialversicherungen sind die Anteile bei der ersten Berechnungsmethode mit je 0,3 Prozent des BIP nahezu identisch. Dies hat damit zu tun, dass die Leistungen kontinuierlich (diskretionär) ausgebaut wurden, während die Einnahmen aufgrund der Ausgestaltung der Finanzierung nur bedingt auf konjunkturelle Schwankungen reagierten. Bei den Kantonen ist der Anteil der automatischen Stabilisatoren nur wenig größer. Da sich in Zukunft die Staatstätigkeit nicht im gleichen Ausmaß ausdehnen kann wie in den Jahren 1951-2007, wird der diskretionäre Impuls aus dem Staatswachstum im Vergleich zu den automatischen Stabilisatoren automatisch schwächer werden.

Tabelle 2 zeigt die diskretionären Impulse des Bundeshaushalts gemäß einer Zusammenstellung von Balastèr (2009). Die Zusammenstellung orientiert sich an den Parlamentsbeschlüssen und nicht an der Datenanalyse. Auch Balastèr (2009) kommt zum Ergebnis, dass die diskretionären Maßnahmen im Vergleich zu den automatischen Stabilisatoren nur einen bescheidenen Effekt auf die Konjunktur ausübten.

Damit liegen die Ergebnisse im Rahmen der internationalen Erfahrungen. Gemäß OECD (2009) sind in der gegenwärtigen Krise die automatischen Stabilisatoren mehr als doppelt so groß wie die kurzfristig beschlossenen Maßnahmen. Interessant ist auch, dass in 95 Prozent aller Rezessionen die automatischen Stabilisatoren ihre Wirkung entfalteten, während diskretionäre Maßnahmen nur in 23 Prozent eine antizyklische Wirkung hatten (IWF 2008). Letztere waren oft schlecht auf den Konjunkturzyklus abgestimmt und wurden vielfach nicht aus Gründen der Konjunkturstabilisierung beschlossen.

\section{Makroökonomische Effekte der Fiskalpolitik}

Nachdem wir die Rezessionsphasen und die fiskalpolitischen Impulse identifiziert haben, stellt sich die Frage der makroökonomischen Effekte. Was waren die Wachstumseffekte, die durch die verfolgte Fiskalpolitik während den rezessiven Phasen ausgelöst wurden? In einem ersten Schritt untersuchen wir diese Frage in einer Event-Analyse. Danach geben Regressionsanalysen systematischere Auskunft über Einflüsse und Effekte. 
Tabelle 3 Auswirkungen der Impulse des Bundeshaushalts (ohne Sozialversicherungen) in Rezessionsjahren (1950-2008)

\begin{tabular}{lccrrrr}
\hline & $\begin{array}{c}\text { Anzahl } \\
\text { Perioden }\end{array}$ & $\begin{array}{c}\text { Drei Jahre } \\
\text { vorher }\end{array}$ & $\begin{array}{c}\text { Ein Jahr } \\
\text { vorher }\end{array}$ & $\begin{array}{c}\text { Jahr der } \\
\text { Krise }\end{array}$ & $\begin{array}{c}\text { Ein Jahr } \\
\text { später }\end{array}$ & $\begin{array}{r}\text { Drei Jahre } \\
\text { später }\end{array}$ \\
\hline$\triangle$ BIP & 7 & 3,3 & 1,8 & $-1,8$ & 2,2 & 2,5 \\
Primärsaldo & 7 & 0,4 & 0,2 & $-0,3$ & $-0,2$ & $-0,1$ \\
$\triangle$ Primärsaldo & 7 & $-0,1$ & $-0,2$ & $-0,5$ & 0,1 & 0,1 \\
$\triangle$ Schulden & 7 & $-0,2$ & 0,6 & 1,1 & 0,5 & 0,2 \\
$\triangle$ Einnahmen & 7 & 0,0 & $-0,1$ & 0,1 & $-0,1$ & 0,1 \\
$\triangle$ Ausgaben & 7 & 0,1 & 0,1 & 0,6 & $-0,2$ & 0,0 \\
$\triangle$ Nominalzins SNB & 7 & 0,6 & 0,5 & $-1,2$ & $-0,4$ & $-0,3$ \\
$\triangle$ Inflation & 7 & 0,8 & 0,7 & $-1,3$ & $-1,6$ & $-0,4$ \\
\hline
\end{tabular}

Quellen: EFV (verschiedene Jahrgänge), BFS (2008), SNB (2009), Seco (2009), eigene Berechnungen

\subsection{Event-Analyse}

Mit der Event-Analyse wollen wir die Dynamik von makroökonomischen Schlüsselvariablen vor, während und nach einer Rezession beobachten. Tabellen 3 und 4 zeigen die Entwicklung von Wachstum, Primärdefiziten, Schulden und nominellen Zinssätzen für die Bundes- wie auch für die Kantonsebene.

Tabelle 3 zeigt, dass die Wirtschaft in den sechs Rezessionen seit 1950 um durchschnittlich 1,8 Prozent pro Jahr geschrumpft ist. Dieser Durchschnitt wird jedoch durch den starken Rückgang von 1975 (BIP-Rückgang von 6,7 Prozent) deutlich verzerrt. In den letzten drei Rezessionen von 1991, 1993 und 2003 lag der Rückgang des BIP jeweils unter einem Prozent. Ein Jahr nach der Rezession lag das Wirtschaftswachstum durchschnittlich bereits wieder bei 2,2 Prozent. Die Veränderungen bei den Einnahmen und Ausgaben deuten auf den Sperrklinkeneffekt gemäß Peacock und Wiseman (1961) hin. In Rezessionen steigen die Ausgaben um durchschnittlich 0,6 Prozent des BIP an. Die Betrachtung der folgenden drei Jahre zeigt, dass die Ausgaben dann auf diesem Niveau verharren. Die Einnahmen nehmen hingegen in den drei Jahren nach einer Rezession jährlich um durchschnittlich 0,1 Prozent des BIP zu. Dass die Einnahmenrückgänge erst ein Jahr verzögert zur Krise auftreten, hat wahrscheinlich mit der zeitlich verzögerten Steuerveranlagung und der früher üblichen zweijährigen Veranlagungsperiode $\mathrm{zu}$ tun. Die Krisen führten erwartungsgemäß zu negativen Primärsaldi und wachsenden Schulden. In den drei Jahren nach der Rezession war der Primärsaldo im Schnitt aber wieder ausgeglichen und die Schulden stiegen nur noch geringfügig stärker an als das BIP (vgl. Reinhart und Rogoff 2009, die für große Krisen im internationalen Vergleich von sehr hohen Schuldenzuwachsraten in der Nachkrisenzeit berichten). Die Geldpolitik reagierte jeweils schnell auf den konjunkturellen Einbruch, indem sie ihren Leitzins um durchschnittlich 120 Basispunkte senkte. Die etwas überraschenden negativen Vorzeichen für die Folgejahre sind vor allem auf die 90er Jahre zurückzuführen. Die SNB hatte ihren Leitzins zwischen 1991 und 1996 von 7 auf 1 Prozent gesenkt, obwohl die Wirtschaft ab 1994 wieder ein positives Wirtschaftswachstum aufwies. Im ähnlichen Ausmaß wie der Leitzins war auch die Inflationsrate gemessen anhand des Landesindexes der Konsumentenpreise (LIK) rückläufig. 


\section{Christoph A. Schaltegger und Martin Weder}

Tabelle 4 Auswirkungen der Impulse der Kantone in Rezessionsjahren (1970-2005)

\begin{tabular}{lccrrrr}
\hline & $\begin{array}{c}\text { Anzahl } \\
\text { Perioden }\end{array}$ & $\begin{array}{c}\text { Drei Jahre } \\
\text { vorher }\end{array}$ & $\begin{array}{c}\text { Ein Jahr } \\
\text { vorher }\end{array}$ & $\begin{array}{c}\text { Jahr der } \\
\text { Krise }\end{array}$ & $\begin{array}{c}\text { Ein Jahr } \\
\text { später }\end{array}$ & $\begin{array}{c}\text { Drei Jahre } \\
\text { später }\end{array}$ \\
\hline$\triangle$ BIP & $\mathbf{2 4 3}$ & $\mathbf{1 , 3}$ & $\mathbf{0 , 5}$ & $-\mathbf{2 , 1}$ & $\mathbf{1 , 0}$ & $\mathbf{1 , 5}$ \\
Prozyklisch & 112 & 1,1 & 0,3 & $-2,1$ & 1,4 & 1,5 \\
Antizyklisch & 131 & 1,5 & 0,7 & $-2,2$ & 0,6 & 1,5 \\
$\triangle$ Primärdefizit & $\mathbf{2 4 3}$ & $\mathbf{0 , 1}$ & $\mathbf{0 , 0}$ & $-\mathbf{0 , 1}$ & $\mathbf{0 , 1}$ & $\mathbf{0 , 1}$ \\
Prozyklisch & 112 & 0,0 & $-0,1$ & 0,5 & 0,0 & 0,0 \\
Antizyklisch & 131 & 0,1 & 0,1 & $-0,7$ & 0,1 & 0,1 \\
Primärdefizit & $\mathbf{2 4 3}$ & $\mathbf{0 , 2}$ & $\mathbf{0 , 2}$ & $\mathbf{0 , 1}$ & $\mathbf{0 , 2}$ & $\mathbf{0 , 3}$ \\
Prozyklisch & 112 & 0,0 & $-0,1$ & 0,5 & 0,4 & 0,5 \\
Antizyklisch & 131 & 0,4 & 0,5 & $-0,2$ & $-0,1$ & 0,1 \\
$\triangle$ Schulden & $\mathbf{2 4 3}$ & $\mathbf{0 , 2}$ & $\mathbf{0 , 4}$ & $\mathbf{1 , 0}$ & $\mathbf{0 , 5}$ & $\mathbf{0 , 1}$ \\
Prozyklisch & 112 & 0,4 & 0,7 & 0,9 & 0,3 & $-0,3$ \\
Antizyklisch & 131 & 0,0 & 0,2 & 1,1 & 0,6 & 0,4 \\
\hline
\end{tabular}

Quellen: EFV (verschiedene Jahrgänge), BFS (2008), eigene Berechnungen ${ }^{9}$

Unsere kantonale Event-Analyse der Rezessionsphasen ist in Tabelle 4 dargestellt. Für die kantonalen Rezessionen ergeben sich zwischen 1971-2005 insgesamt 243 Beobachtungen. Dabei wird deutlich, dass sich die Fiskalpolitik der Kantone in Rezessionsjahren nicht wesentlich von derjenigen über den gesamten Konjunkturzyklus unterscheidet. Antizyklische und prozyklische Maßnahmen sind in etwa gleich häufig (54 Prozent der Fälle antizyklisch, 46 Prozent der Fälle prozyklisch). Diese Anteile bewegen sich damit auf ähnlicher Höhe wie die eingangs zitierten Analysen von Frick und Lampart (2004) und Frey (2007), welche jedoch nicht zwischen Rezessionen und Expansionsphasen differenzieren. Bis Ende der 90er-Jahre ist bei der Finanzpolitik in Rezessionsphasen kein Trend in eine Richtung ersichtlich. In den letzten Jahren verfolgten die Kantone aber ähnlich wie der Bund vermehrt eine antizyklische Fiskalpolitik (zwei Drittel der Fälle im Zeitraum 2001-2005). Dabei zeigt sich im Krisenjahr ein ausgeprägter Unterschied in den Primärsalden zwischen jenen Kantonen, die eine antizyklische Fiskalpolitik verfolgten, im Vergleich zu jenen, die eine prozyklische Fiskalpolitik aufweisen.

Interessant ist weiter, dass das Ausmaß der Rezession kaum einen Einfluss darauf hat, ob die Kantone kurzfristig zusätzliche Ausgaben oder Steuersenkungen beschlossen haben. Im Durchschnitt ist der Rückgang des Volkseinkommens sowohl bei einer anti- wie auch bei einer prozyklischen Fiskalpolitik im Jahr der Krise nahezu identisch (-2,1 bzw. -2,2 Prozent). Das durchschnittliche jährliche Wachstum des Volkseinkommens ist mit 1,5 Prozent in den drei Jahren nach der Krise ebenfalls in beiden Fällen gleich hoch. Damit zeigt sich eine prozyklische Politik nicht als ausgeprägt wachstumsfeindlich. Weil sich Kantone, die eine prozyklische Fiskalpolitik verfolgten, in den Jahren vor der Krise oftmals mit Haushaltsdefiziten und steigenden Schulden konfrontiert sahen, liegt die Vermutung nahe, dass die Entscheidung zu Einsparungen oder Steuererhöhungen während Rezessionen nicht gezielt, sondern primär aus haushalterischer Notwendigkeit geschah. Demgegenüber konnten Kantone, welche

9. Es bestehen zwischen Primärdefizit und Veränderung des Primärdefizits kleine Rundungsdifferenzen. 


\section{Fiskalpolitik als antizyklisches Instrument?}

vor der Krise einen strukturell ausgeglichenen Haushalt hatten, eher mit einer antizyklischen Fiskalpolitik der Krise entgegenwirken. Dieser Befund ist im Einklang mit der Event-Studie des IWF (2008), die in der jeweiligen Verschuldungshöhe eines Landes einen wichtigen Bestimmungsgrund fiskalpolitischer Impulse sehen.

\subsection{Regressionsanalyse}

Die Event-Analyse zeigt die Dynamik verschiedener wichtiger makroökonomischer Variablen. Die Analyse sagt aber nichts über Beziehungen und Effekte zwischen den Variablen. In der Regressionsanalyse wollen wir neben dem Fiskalimpuls verschiedene andere Faktoren wie die Geldpolitik und andere Determinanten der gesamtwirtschaftlichen Nachfrage simultan betrachten, um die Wirkung auf das reale Wachstum der Volkswirtschaft zu evaluieren.

Ein wesentliches Problem stellt dabei die Endogenität des Fiskalimpulses mit dem volkswirtschaftlichen Output dar: Fiskalpolitik und Wirtschaftswachstum beeinflussen einander gegenseitig, so dass es methodisch schwierig zu bestimmen ist, welcher Effekt worauf zurückgeführt werden kann. Eine Möglichkeit, den Einfluss der Schocks der Fiskalpolitik auf das reale Wachstum zu schätzen, besteht mit Vektorautoregressionen (VAR Modellen). Das Problem der Endogenität wird dabei durch jeweils um zwei Perioden verzögerte Variable zumindest entschärft. VAR Modelle haben den Vorteil, dass sie im Gegensatz zu Fallstudien den systematischen Vergleich über verschiedene Staaten oder Gebietskörperschaften zulassen. Sie ermöglichen zudem, die Einflussfaktoren zu quantifizieren und Prognosen abzuleiten. Neben dem Endogenitätsproblem sind allerdings die Berücksichtigung der wirtschaftlichen Situation sowie die bereits zu Beginn erläuterte Aufteilung in automatische und diskretionäre Maßnahmen schwierig und zu einem bestimmten Teil auch subjektiv. Der geschätzte Effekt eines Stimulus kann weiter dadurch verzerrt werden, dass Veränderungen auf der Einnahmenund Ausgabenseite je nach konkreter Maßnahme nicht die gleiche Wirkung haben. So spielt es eine Rolle, für welche Sachgebiete zusätzliche Ausgaben beschlossen wurden (Transfers oder Investitionen) oder welche Bevölkerungsgruppen von Steuersenkungen profitieren (unterschiedliche Konsumneigung).

Zur Analyse der Wirkung der Fiskalpolitik des Bundes auf das Wachstum, lehnen wir uns an den von Blanchard und Perotti (2002) entwickelten Ansatz eines VAR mit Jahresdaten für die Periode 1951-2007. ${ }^{10}$ Unsere VAR-Spezifikation lautet wie folgt:

$$
\mathrm{Y}_{\mathrm{t}}=\mathrm{A}(\mathrm{L}) \mathrm{Y}_{\mathrm{t}-1}+\mathrm{U}_{\mathrm{t}}
$$

wobei $Y_{t}=\left(X_{t}, T_{t}, G_{t}, R_{t}, I_{t}, W_{t}\right)$ ein Vektor endogener Variablen darstellt. $A(L)$ ist ein autoregressiv verzögertes Polynom, das die Beziehung der Koeffizienten zur abhängigen Variablen beschreibt. X steht für das reale Wirtschaftswachstum, $\mathrm{T}$ für die realen Staatseinnahmen, $\mathrm{G}$ für die realen Staatsausgaben, $\mathrm{R}$ für

10. Viele Studien zur Wirkung von Fiskalimpulsen verwenden Quartalsdaten (beispielsweise auch Blanchard und Perotti 2002 oder de Castro und Hernandez de Cos 2007). Aufgrund der besseren Datenqualität von Jahresdaten im Falle der Schweiz haben wir uns gegen die Verwendung von Quartalsdaten entschieden. 


\section{Christoph A. Schaltegger und Martin Weder}

Tabelle 5 Likelihood Test und Informationskriterien

\begin{tabular}{lcccccc}
\hline $\begin{array}{l}\text { Anzahl } \\
\text { Perioden }\end{array}$ & $\begin{array}{c}\text { Log } \\
\text { likelihood }\end{array}$ & LR & p-Wert & AIC & HQIC & SBIC \\
\hline 0 & 187,853 & & & $-6,106$ & $-5,779$ & $-5,258$ \\
1 & 189,924 & $4,143^{*}$ & 0,042 & $-6,145^{*}$ & $-5,804^{*}$ & $-5,261^{*}$ \\
2 & 190,295 & 0,742 & 0,389 & $-6,122$ & $-5,767$ & $-5,201$ \\
3 & 191,257 & 1,923 & 0,166 & $-6,121$ & $-5,751$ & $-5,163$ \\
\hline
\end{tabular}

Bemerkung: 54 Beobachtungen für den Bund (1954-2007). - LR: Likelihood Ratio Test, AIC: Akaikes Informationskriterium, HQIC: Hannan-Quinn Informationskriterium, SBIC: Schwarz-Bayes Informationskriterium. Das gewählte Modell entspricht weitgehend Schätzgleichung (2), außer dass drei statt zwei Lags verwendet wurden.

Quelle: Eigene Berechnungen mit Stata

den Nominalzinssatz, I für die privaten Investitionen und $W$ für das reale Weltwirtschaftswachstum. Die Anzahl der verzögerten Werte (Lags) wurde wie in der Analyse des IWF auf zwei gesetzt, um unkorrelierte Residuen zu erhalten. Wie Tabelle 5 zeigt, wäre es gemäß Likelihood Test sogar erlaubt gewesen, nur mit einer Verzögerung von einem Jahr zu rechnen.

Der Vektor $U_{t}=\left(u^{x}, u^{t}, u^{g}, u^{r}{ }_{t}, u^{w}{ }_{t}\right)$ beschreibt die unkorrelierten Residuen der reduzierten Form. Analog zu Blanchard und Perotti (2002) wurden verschiedene Tests (Augmented Dickey-Fuller Test, Phillips-Peron Test, KPSS Test) zur Überprüfung der Stationarität durchgeführt (siehe Anhang D). Stationarität bedeutet, dass eine Zeitreihe zu jedem Zeitpunkt den gleichen Erwartungswert und die gleiche Varianz aufweist. Die meisten ökonomischen Variablen sind aber nicht stationär, sondern sie unterliegen häufig einem bestimmten Trend. Werden Variablen mit einem Trend dennoch verwendet, so kann dies zu verzerrten Ergebnissen führen. Eine Möglichkeit, dieses Problem zu entschärfen besteht darin, mit ersten Differenzen $\left(\Delta \mathrm{x}_{\mathrm{t}}=\mathrm{x}_{\mathrm{t}}-\mathrm{x}_{\mathrm{t}-1}\right) \mathrm{zu}$ arbeiten. Bei der Verwendung von Niveau-Daten zeigt sich, dass Trend-Stationarität bei den Primärausgaben und beim BIP pro Kopf verworfen werden können. Im Falle der Einnahmen kann die Trend-Stationarität nicht bei allen Tests abgelehnt werden (Anhang D). Wir haben uns daher für die Verwendung von ersten Differenzen und nicht von Niveaus entschieden. Anstelle der Höhe der Staatsausgaben in Prozent des BIP wurde somit mit der Veränderung der Staatsausgaben gegenüber dem Vorjahr in Prozent des BIP gerechnet. In diesem Fall kann die Nullhypothese, dass eine Einheitswurzel vorliegt, unabhängig vom gewählten Test deutlich verworfen werden. Die Normalverteilung der Residuen kann gemäß Shapiro-Wilk W Test mit Werten um 0,96 ebenfalls nicht abgelehnt werden. Das Korrelationsdiagramm (Anhang C.1) zeigt zudem, dass wir bei den verwendeten Variablen kaum Probleme der Multikollinearität haben. Die Ergebnisse sollten daher nicht verzerrt sein. Bei der Identifikation der Fiskalschocks folgen wir dem IWF (2008). Die elastizitäts-basierten Fiskalimpulse - automatisch, wie auch diskretionär - wurde bereits in Gleichung 2 und 3 ermittelt.

Wir verwenden vier verschiedene Modelle. Die ersten beiden Modelle unterscheiden sich durch die abhängige Variable. Im ersten Fall wurde das reale BIP Wachstum, im zweiten Fall das reale Wachstum des BIP pro Kopf verwendet. Das dritte Modell rechnet die Ausgaben- und Einnahmenveränderungen der Arbeitslosenversicherung (ALV) zum Bundeshaushalt hinzu. Der Impuls wurde dabei vollständig den automatischen Stabilisatoren zugerechnet. Im vierten Modell 


\section{Fiskalpolitik als antizyklisches Instrument?}

wurden die konsolidierten Einnahmen- und Ausgabenveränderungen der vier staatlichen Sozialversicherungen AHV, IV, ALV und EO dem Bundeshaushalt zugerechnet. Der Impuls der Sozialversicherungen wurde wiederum als vollständig automatisch definiert. Die Ergebnisse sind in Tabelle 6 festgehalten.

In allen vier Modellen hatten die automatischen Stabilisatoren im Jahr der Rezession einen positiven und statistisch signifikanten Einfluss auf das Wachstum, während der Effekt von diskretionären Maßnahmen negativ, wenn auch nicht signifikant war. Letztere hatten hingegen mit einer Verzögerung von zwei Jahren überraschenderweise einen stark positiven Effekt auf das Wachstum, was auf eine ausgeprägt prozyklische Wirkung hinweist. Signifikant waren zudem das Wachstum der Vorperiode, das Bevölkerungswachstum, die Veränderung des Leitzinses der Nationalbank, die Investitionen und das Wachstum der Weltwirtschaft. Ausgabenveränderungen, die außerhalb von Rezessionen stattfanden, hatten hingegen keinen signifikanten Einfluss.

Die Ergebnisse decken sich damit mehrheitlich mit jenen des IWF (2008) im Falle von Länderanalysen. Der IWF (2008) erklärt sich die häufig insignifikanten Resultate der Fiskalimpulse mit der geringen Anzahl an Beobachtungen und der damit verbundenen ungenügenden Variation innerhalb der Stichprobe. Er verwendet jedoch Quartalsdaten und kommt damit auf über 140 Beobachtungen pro Land, während für unsere Analyse unter Berücksichtigung der verzögerten Variablen um zwei Perioden nur 55 Beobachtungen zur Verfügung stehen.

Voraussetzung für eine konsistente VAR-Analyse ist, dass wir über den gesamten Zeitraum von 1951 bis 2007 über eine stabile Struktur verfügen. Das Ende des Bretton Woods Regimes mit fixen Wechselkursen zu Beginn der 70er Jahre stellt diese Stabilität in Frage. Es ist plausibel, dass die Wirkung der Fiskalpolitik in einer kleinen offenen Volkswirtschaft wie der Schweiz durch eine solche Veränderung spürbar beeinflusst wurde. Wir haben daher verschiedene Tests auf einen möglichen Strukturbruch durchgeführt (siehe Tabellen 7 und 8).

Sowohl der Clemente-Montañes-Reyes-Test als auch der Zivot-Andrews-Test bestätigen, dass zu Beginn der 70er Jahre tatsächlich ein Bruch festzustellen ist. Die entsprechende t-Statistik liegt mit Werten von -4.012 bzw. -6.752 jeweils deutlich über der kritischen Schwelle. Als Wendepunkt wird das Jahr 1973 bzw. 1974 ausgewiesen. Dies ist auch mit der Faktenlage übereinstimmend: Im Januar 1973 intervenierte die Schweizerische Nationalbank SNB erstmals nicht mehr auf dem Dollarmarkt zur Einhaltung der Franken-Dollar Parität und kommunizierte dies auch so (SNB 2007).

Die Gleichungen (5) bis (7) in Tabelle 6 zeigen die Ergebnisse dieser Analyse. Für die ersten beiden Schätzgleichungen wurde eine Dummy Variable verwendet, welche für die Zeit ab 1973 den Wert 1 annimmt. ${ }^{11}$ Die Dummy Variable ist statistisch signifikant, das Wirtschaftswachstum war ab 1973 deutlich tiefer als zuvor. Für die Gleichung (7) wurde eine Schätzung nur für die Jahre 1973 bis 2007 durchgeführt. ${ }^{12}$ Gemäß KPSS-Test (siehe Anhang D) kann Trendstationarität für die ersten Differenzen auch im Falle der zerlegten Zeitreihe nicht abgelehnt werden. Der kritische Wert von 0.119 wird nicht erreicht. Die bisherigen Ergebnisse erweisen

11. Als sinnvolles und einfaches Mittel zur Darstellung von Strukturbrüchen werden in der Literatur oft Dummy Variabeln vorgeschlagen (vgl. z.B. Kirchgässner und Wolters 2006).

12. Eine analoge Analyse für den Bundeshaushalt für die Zeit von 1951-1972 war aufgrund der mangelnden Anzahl an Beobachtungen nicht möglich. 


\section{Christoph A. Schaltegger und Martin Weder}

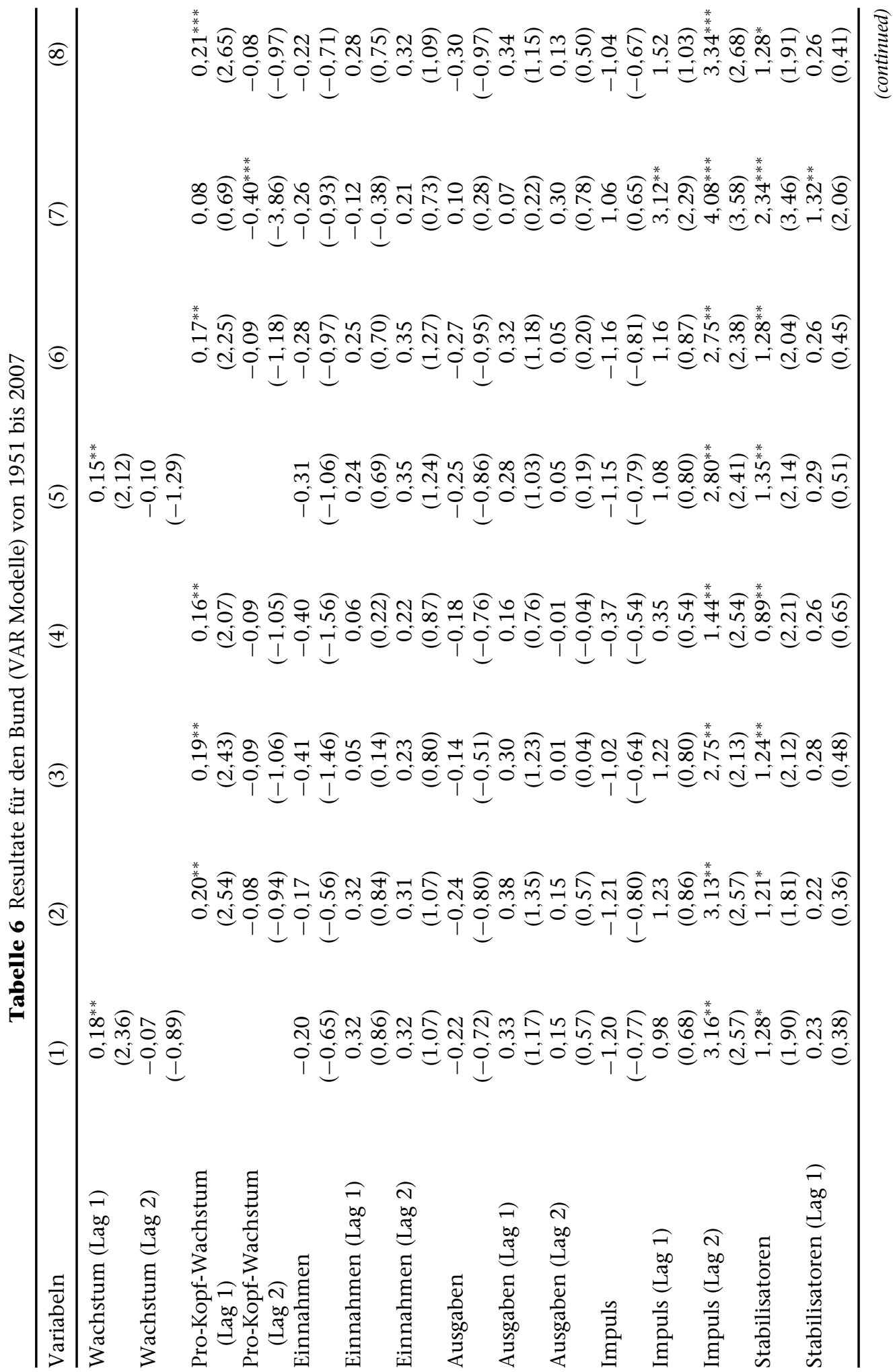


Fiskalpolitik als antizyklisches Instrument?

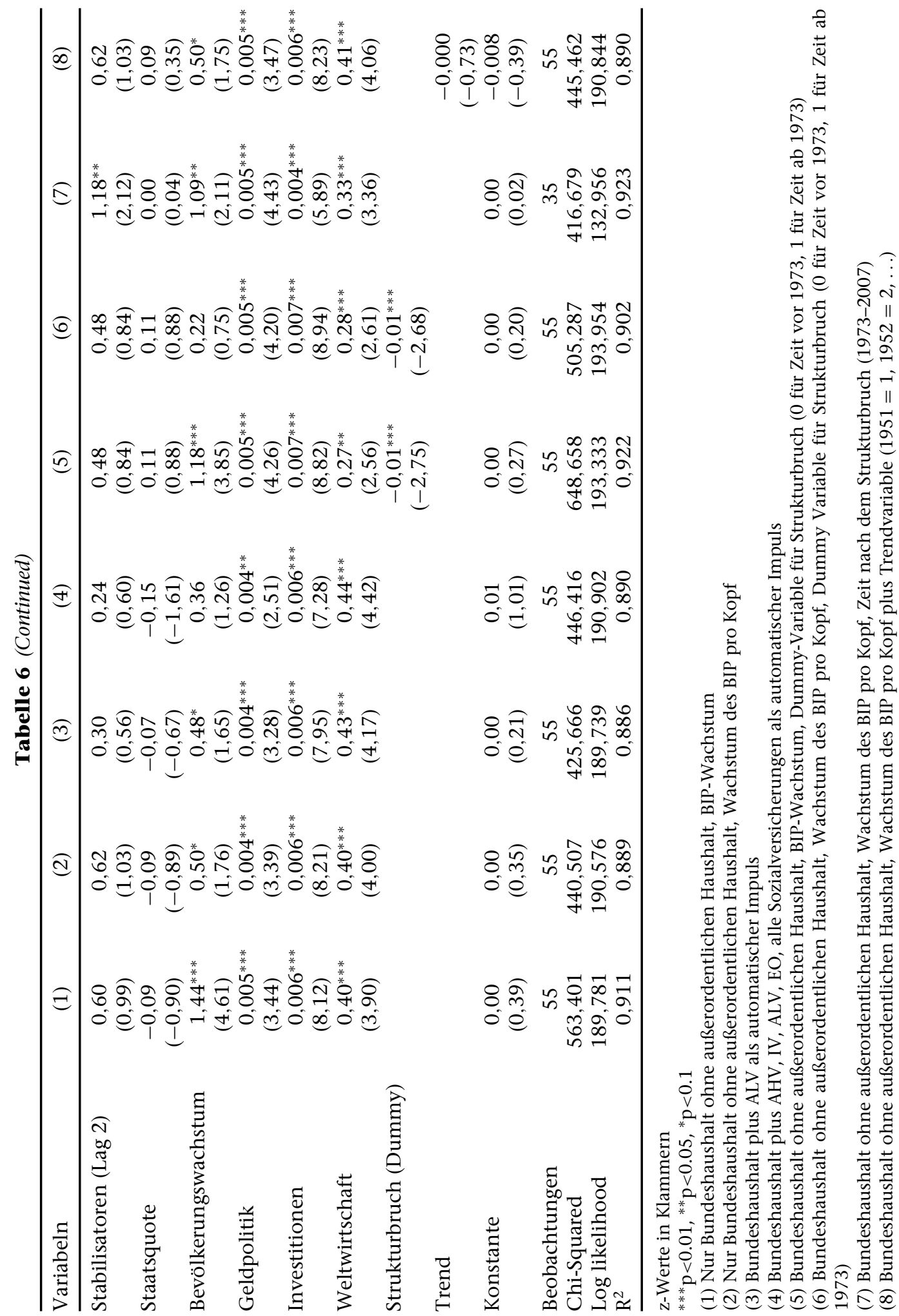




\section{Christoph A. Schaltegger und Martin Weder}

Tabelle 7 Ergebnis des Clemente-Montañes-Reyes Einheitswurzel Tests

\begin{tabular}{lcr}
\hline & \multicolumn{2}{c}{ Clemente-Montañes-Reyes } \\
Einheitswurzel Test
\end{tabular}

Quelle: Eigene Berechnungen mit Stata

Tabelle 8 Ergebnis des Zivot-Andrews Einheitswurzel Tests

\begin{tabular}{llc}
\hline Wachstum des BIP pro Kopf & \multicolumn{2}{c}{ Zivot-Andrews Einheitswurzel Test } \\
\hline Minimale t-Statistik & $-6,752$ & (Jahr 1974) \\
Kritischer Wert (1\% Niveau) & $-5,430$ & \\
Kritischer Wert (5\% Niveau) & $-4,800$ & \\
\hline
\end{tabular}

Quelle: Eigene Berechnungen mit Stata

sich insgesamt also als robust: Das Wirtschaftswachstum wird signifikant positiv beeinflusst durch das Niveau der Investitionen, die Geldpolitik und das Wachstum der Weltwirtschaft. Diskretionäre Impulse haben erst mit einer Verzögerung eine spürbare Wirkung, demgegenüber wirken die automatischen Stabilisatoren bereits im Jahr der Rezession. Ein langfristiger Wachstumstrend über den gesamten Zeitraum konnte nicht festgestellt werden. Die entsprechende Trend-Variable erwies sich als nicht signifikant (Gleichung 8).

\subsection{Impuls-Antwort Analyse}

Zur Quantifizierung der Impuls-Antwort-Reaktion haben wir zwei einfache SVAR-Modelle mit fünf bzw. sechs Variablen geschätzt (Veränderung der Geldpolitik, diskretionäre Fiskalpolitik, automatische Stabilisatoren, Wachstum der Investitionen, Weltwirtschaftswachstum sowie reales Pro-Kopf-Einkommen). SVARModelle bieten gegenüber VAR Modellen den Vorteil, dass zusätzliche kurz- und langfristige Einschränkungen bzw. Nebenbedingungen formuliert werden können. Wir haben für das SVAR-Modell folgende Einschränkungen unterstellt:

- Pro-Kopf-Wachstum: Kann von allen anderen Variablen beeinflusst werden.

- Weltwirtschaftswachstum: Wird von keiner anderen Variable beeinflusst.

- Investitionen: Alle Variablen haben einen potenziellen Einfluss.

- Fiskalpolitik: Außer der Investitionen haben alle anderen Faktoren einen Einfluss.

- Geldpolitik: Pro-Kopf-Wachstum, Fiskalpolitik und Weltwirtschaftswachstum beeinflussen die Veränderung der Geldpolitik, die Investitionen hingegen nicht.

Für das 5-SVAR Modell wurden die automatischen Stabilisatoren nicht berücksichtigt. Abbildung 5 mit der Impulse-Antwort-Analyse hält die Ergebnisse 


\section{Fiskalpolitik als antizyklisches Instrument?}

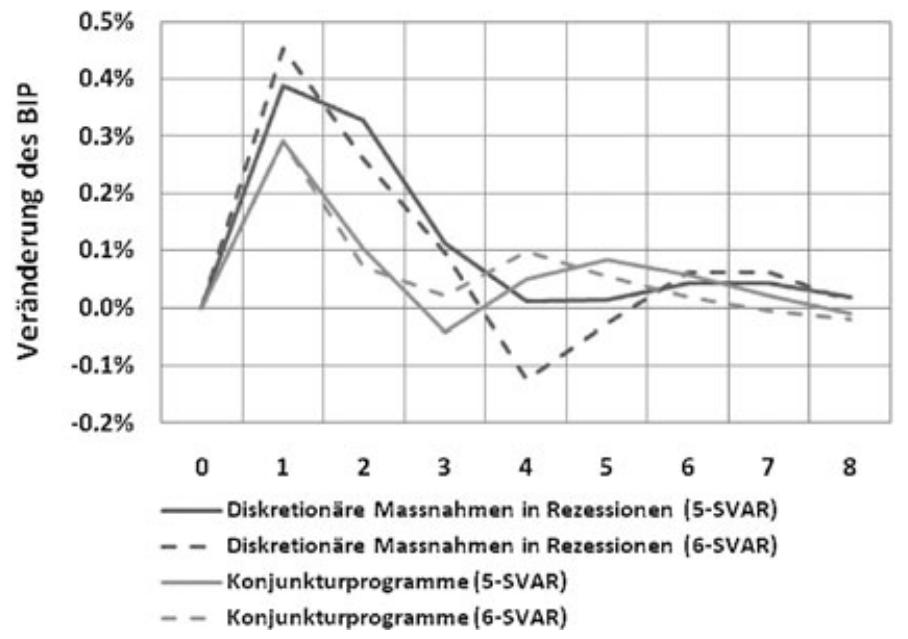

Abbildung 5 Einfluss diskretionärer Maßnahmen und Konjunkturprogramme auf das Wirtschaftswachstum

Quelle: Eigene Berechnungen anhand von SVAR Modellen mit STATA

fest. Im 5-SVAR Modell beträgt der Einfluss der diskretionären Fiskalpolitik auf das Wachstum ein Jahr nach der Rezession 0,39 Prozent des BIP. Da der Effekt im Jahr der Rezession bei null liegt, beträgt der kurzfristige Multiplikator 0,39. Der langfristige Multiplikator liegt bei 0,96. Betrachtet man isoliert die in der Vergangenheit vom Bund verabschiedeten Konjunkturprogramme, welche teilweise außerhalb von Rezessionen beschlossen wurden, so liegt der Einfluss bei 0,29 Prozent. Der gesamte Multiplikator liegt in diesem Fall bei 0,55. Im 6-SVAR Modell ist der Effekt ein Jahr später vergleichbar mit dem 5-SVAR Modell: Diskretionäre Maßnahmen während Rezessionen erhöhen das BIP um 0,45 Prozent, nimmt man nur die Konjunkturprogramme gemäß der Zusammenstellung von Balastèr ergibt sich ein Wert um 0,29 Prozent. Die langfristigen Multiplikatoren liegen bei 0,79 bzw. 0,53. Diese Zahlen sind vergleichbar mit den Ergebnissen des IWF (2008). Dieser hatte einen langfristigen Multiplikator von 0,5 berechnet, wobei die Industrieländer deutlich besser abschnitten als die Entwicklungsländer, welche teilweise negative Multiplikatoren aufwiesen. Im Jahr der Rezession lagen die Multiplikatoren der Industrieländer im Schnitt bei 0,1 Prozent.

\subsection{Regressionsanalyse für die kantonale Fiskalpolitik}

Die Schweiz ist ein ausgeprägt föderalistischer Staat mit starker Finanzautonomie der Kantone, die durch Artikel 47 bis 49 der Bundesverfassung gesichert wird. Mit einem Ausgaben- und Einnahmenanteil von knapp $70 \%$ an den Gesamtausgaben bzw. den Gesamteinnahmen, nehmen die Kantone und Gemeinden finanzwirtschaftlich eine bedeutende Stellung ein. Auch wenn, wie eingangs erwähnt, der verfassungsrechtliche Auftrag zur Stabilisierungspolitik dem Bund zufällt, ist es wichtig, dass die Kantons- und Gemeindehaushalte die Bundespolitik nicht neutralisieren. Im Folgenden untersuchen wir deshalb die makroökonomischen Effekte der von Kantonen verfolgten Fiskalpolitik. In 


\section{Christoph A. Schaltegger und Martin Weder}

Anlehnung an die Studie des IWF (2008) benutzen wir für unsere PanelAnalyse der 26 Kantone über den Zeitraum 1971 bis 2005 einen Arellano-Bond Schätzer (Arellano und Bond 1991). Dieser Ansatz eignet sich besonders für Datensätze mit vergleichsweise vielen Analyseobjekten (hier: Kantone) und kurzen Beobachtungsperioden (Mileva 2007). Unser Datensatz besteht aus insgesamt 901 Beobachtungen (26 Kantone über 35 Jahre). Damit erfüllen wir wie die Studie des IWF (2008) die Kriterien für einen Arellano-Bond-Schätzer nicht vollumfänglich und können eine Verzerrung der Ergebnisse nicht ausschließen. Die entsprechenden Tests zeigen jedoch, dass die Schätzungen konsistent sind. Der Arellano-Bond Test lieferte keine Hinweise, dass die Störterme in der Niveaugleichung autokorreliert wären (vgl. Tabelle 9). Ebenso können anhand des Sargan Test überidentifizierbare Restriktionen abgelehnt werden (p-Wert liegt stets bei null). Die gewählten Instrumente erscheinen daher geeignet. Wäre dies nicht der Fall gewesen, so wäre anstelle des Arellano-Bond Schätzverfahren ein SystemGMM-Schätzer nach Blundell und Bond (1998) die bessere Wahl gewesen. Ein weiterer Vorteil dieses Schätzverfahrens besteht darin, dass es sich für Regressionen eignet, bei welchen die unabhängigen Variablen nicht strikt exogen sind, sich also auf vergangene und gegenwärtige Ausprägungen des Fehlerterms auswirken können. Außerdem werden mit fixen Effekten die kantons- und zeitspezifischen Charakteristiken aufgefangen und die Heteroskedastie und Autokorrelation in den Daten gemildert (Roodman 2008).

Die zu erklärende Variable beschreibt das Wachstum des Pro-Kopf-Einkommens $\left(\mathrm{w}_{\mathrm{t}}\right)$. Wir schätzen folgende Gleichung:

$$
\begin{aligned}
\mathrm{w}_{\mathrm{t}}= & \mathrm{c}+\alpha_{1} \mathrm{w}_{\mathrm{t}-1}+\alpha_{2} \mathrm{w}_{\mathrm{t}-2}+\alpha_{3} \mathrm{r}_{\mathrm{t}}+\alpha_{4} \mathrm{r}_{\mathrm{t}-1}+\alpha_{5} \mathrm{r}_{\mathrm{t}-2}+\alpha_{6} \mathrm{p}_{\mathrm{t}}+\alpha_{7} \mathrm{p}_{\mathrm{t}-1} \\
& +\alpha_{8} \mathrm{p}_{\mathrm{t}-2}+\alpha_{9} \text { disk }_{\mathrm{t}}+\alpha_{10} \text { disk }_{\mathrm{t}-1}+\alpha_{11} \text { disk }_{\mathrm{t}-2}+\alpha_{12} \text { auto }_{\mathrm{t}}+\alpha_{13} \text { auto }_{\mathrm{t}-1} \\
& +\alpha_{14} \text { auto }_{\mathrm{t}-2}+\alpha_{15} \text { bev }_{\mathrm{t}}+\alpha_{16} \text { mon }_{\mathrm{t}}+\alpha_{17} \text { staat }_{\mathrm{t}}+\alpha_{18} \text { niveau }_{\mathrm{t}} \\
& +\alpha_{19} \text { schulden }_{\mathrm{t}} \text { mit c als Konstante sowie einem Störterm } \varepsilon_{\mathrm{t}} .
\end{aligned}
$$

Die verwendeten erklärenden Variablen und deren Reihenfolge sind:

- $\quad$ w: Das Wachstum des Pro-Kopf-Einkommens der Perioden t, t-1 und t-2;

- r: Die Veränderungen der Kantonseinnahmen in Prozent des Volkseinkommens der laufenden Periode $t$ sowie der beiden Vorperioden $t-1$ und $\mathrm{t}-2$;

- p: Die Veränderungen der kantonalen Primärausgaben in Prozent des Volkseinkommens der laufenden Periode $t$ sowie der beiden Vorperioden $t-1$ und $\mathrm{t}-2$;

- disk: Diskretionäre Fiskalpolitik in Rezessionen (Ausgabenerhöhungen, Steuersenkungen) in Prozent des kantonalen Volkseinkommens für die Perioden $\mathrm{t}, \mathrm{t}-1$ und $\mathrm{t}-2$;

- auto: Das Ausmaß der automatischen Stabilisatoren (konjunkturell bedingte Mindereinnahmen und Ausgabenerhöhungen) während Rezessionen in Prozent des kantonalen Volkseinkommens für die Perioden $t, t-1$ und $t-2$;

- bev: Die Zunahme des jeweiligen Bevölkerungswachstums in Prozent in der Periode t;

- mon: Die Geldpolitik, ausgedrückt durch die Veränderung des Leitzinssatzes in Prozentpunkten gegenüber dem Vorjahr in der Periode t; 


\section{Fiskalpolitik als antizyklisches Instrument?}

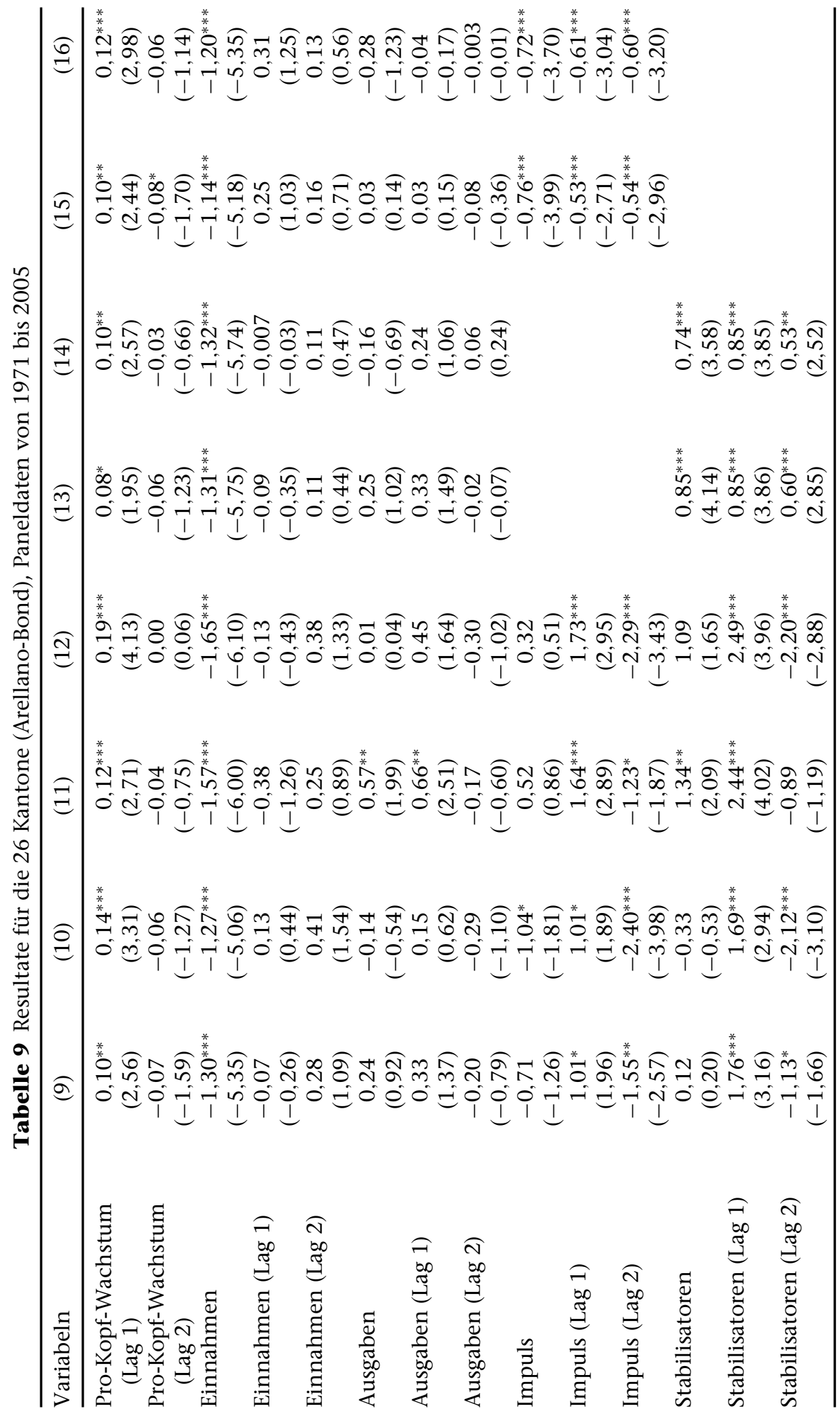


Christoph A. Schaltegger und Martin Weder

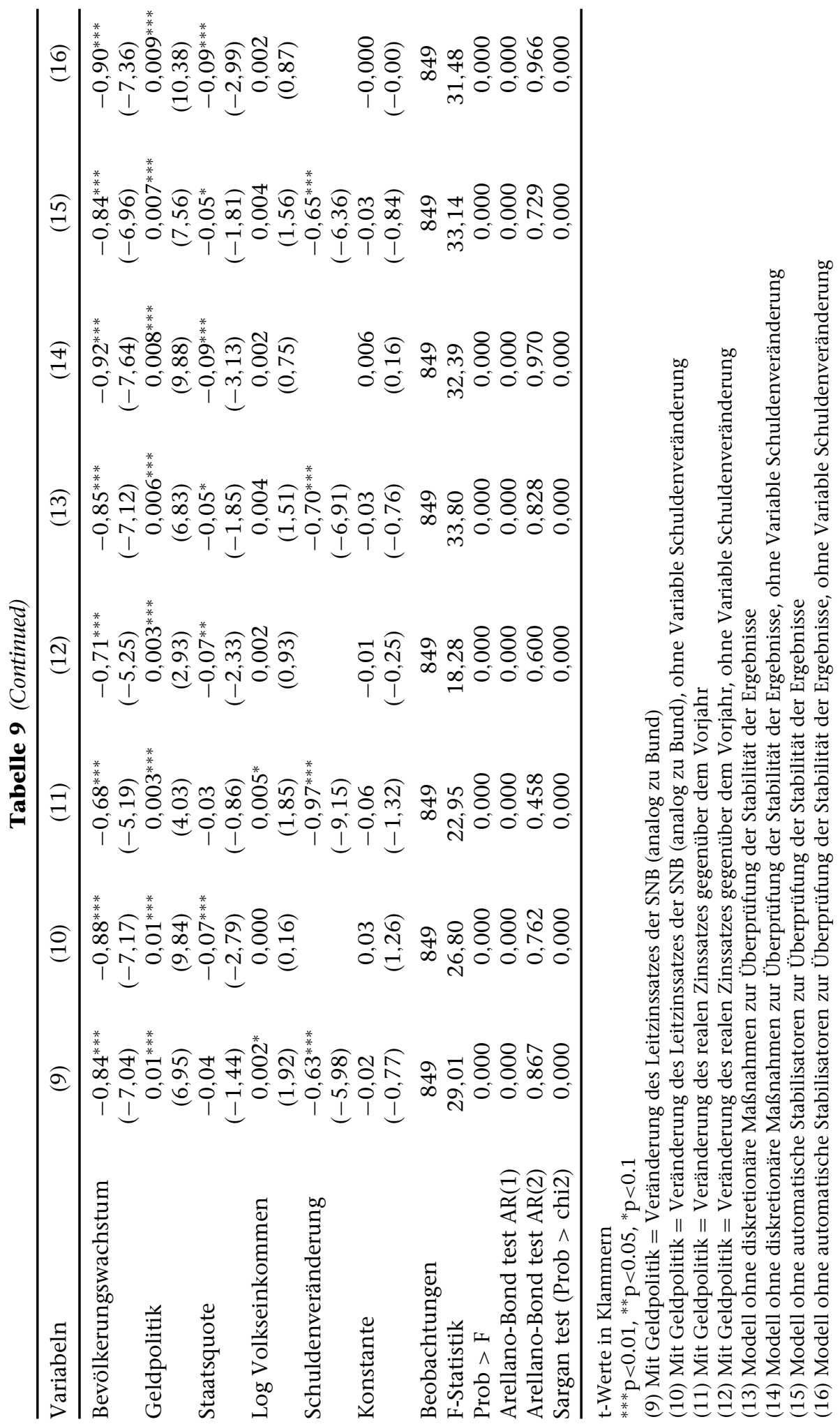




\section{Fiskalpolitik als antizyklisches Instrument?}

- $\quad$ staat: Die Staatsquote, ausgedrückt durch die Gesamtausgaben in Prozent des kantonalen Volkseinkommens in der Periode t;

- niveau: Das Niveau des kantonalen Pro-Kopf-Einkommens um den in der Wachstumstheorie postulierten Aufholeffekt zu überprüfen, wonach Staaten mit hohen Pro-Kopf-Einkommen weniger stark wachsen als solche mit niedrigerem Einkommen in $\mathrm{t}$;

- $\quad$ schulden: Die Auswirkungen der Schulden, ausgedrückt durch die Zunahme der Schulden in Prozent des kantonalen Volkseinkommen in der Periode $t$.

Zudem wurden bei der Staatsquote und der Höhe des Pro-Kopf-Einkommens die Werte der Vorperiode und der Vorvorperiode als Instrumentalvariablen zur Adressierung des Endogenitätsproblems aufgenommen. Die Ergebnisse sind in Tabelle 9 festgehalten. Der Sargan-Test ist hoch signifikant und zeigt, dass die Instrumente nicht mit dem Störterm korrelieren. Das Problem der Multikollinearität besteht gemäß Anhang C.2 im Falle der Stabilisatoren und der Impulse.

Wie die Gleichungen (13) und (15) im Vergleich $\mathrm{zu}$ (9) und (11) zeigen, ändert sich im Falle der diskretionären Impulse etwas an der qualitativen Aussage: Während der Einfluss der Stabilisatoren robust bleibt, nährt der Vorzeichenwechsel bei den diskretionären Impulsen Zweifel an deren Wirkung. Gleichung (11) verwendet eine alternative Variable für die Geldpolitik zur Überprüfung der Stabilität der Ergebnisse. Anstelle der Veränderung des Leitzinssatzes der SNB gegenüber dem Vorjahr wurde die Veränderung des realen Zinssatzes (Leitzinssatz abzüglich der Inflationsrate) verwendet.

Die Resultate lassen sich wie folgt interpretieren: Wie bereits auf Bundesebene erhöht die Wachstumsrate des Pro-Kopf-Einkommens aus der Vorperiode die Wachstumsrate pro Kopf signifikant. Das Bevölkerungswachstum wirkt sich negativ auf das Einkommen pro Kopf aus. Der Effekt der Geldpolitik ist hoch signifikant, wenn auch quantitativ gering. Im Gegensatz zur Analyse auf der Bundesebene haben Veränderungen auf der Einnahmen- und Ausgabenseite einen Einfluss auf das Wachstum des Pro-Kopf-Einkommens. Eine Erhöhung der Einnahmen dämpft das Wachstum bereits in der aktuellen Periode - der Effekt ist hoch signifikant und größer als bei einer entsprechenden Veränderung auf der Ausgabenseite. Veränderungen auf der Ausgabenseite in der aktuellen und der vorherigen Periode erhöhen das Pro-Kopf-Wachstum nur in Gleichung (11). In Rezessionen beschlossene diskretionäre Maßnahmen wirken erst verzögert. Heute beschlossene Maßnahmen wirken sich erst im nächsten Jahr statistisch signifikant auf das Wachstum aus. Dieser Effekt ist einerseits nicht robust wie Gleichungen (15) und (16) zeigen und zudem geringer als jener der automatischen Stabilisatoren. Je nach Modell wirken Letztere bereits in der aktuellen Periode (vgl. Gleichungen (11), (13) und (14)). Im folgenden Jahr verstärkt sich der Impuls. Die Wirkung der Fiskalimpulse läuft nach etwa zwei Jahren aus. Das Niveau des kantonalen Volkseinkommens ist ebenfalls signifikant, hat jedoch nicht das erwartete Vorzeichen. In der Tat sind jene Kantone, welche 1970 ein überdurchschnittlich hohes Pro-Kopf-Einkommen aufwiesen, deutlich stärker gewachsen als die anderen. Die Unterschiede zwischen den Kantonen sind demnach im Laufe der Jahre größer geworden. Während 1970 alle Kantone ein Pro-KopfEinkommen zwischen 24`000 und 48`000 Franken aufwiesen, lag im Jahr 2005 die Spannweite zwischen 34`000 und 91`000 Franken. Der signifikant negative Effekt der Schuldenerhöhung auf das Wirtschaftswachstum deutet darauf hin, 


\section{Christoph A. Schaltegger und Martin Weder}

dass die Wirtschaftssubjekte die Folgen der Staatsverschuldung erkennen. Ihnen ist bewusst, dass sie letztlich deren Kosten, z.B. in Form von Steuererhöhungen, tragen müssen und halten sich deshalb bei steigenden Schulden mit Konsumausgaben und Investitionen zurück.

Der Arellano-Bond Test für Autokorrelation der Residuen liefert keine problematischen p-Werte. Die Nullhypothese „keine Autokorrelation“ kann zwar im Fall der ersten Ordnung in allen Gleichungen abgelehnt werden (p-Wert von null). Wichtiger ist aber die Autokorrelation zweiter Ordnung, da keine Autokorrelation der Störterme in der Niveaugleichung auftreten darf: Mit p-Werten zwischen 0,458 und 0,867 kann Autokorrelation zweiter Ordnung abgelehnt werden.

Obschon die bisherigen Tests sowie die Korrelationskoeffizienten der einzelnen Variablen keinen Anlass zur Sorge gegeben haben, wurde zur Sicherheit in den Gleichungen (10), (12), (14) und (16) die Variable der Schuldenveränderung in Prozent des BIP gegenüber dem Vorjahr weggelassen, da diese zu Multikollinearität mit den unverzögerten Einnahmen und Ausgaben führen könnte. ${ }^{13}$ Zwischen 1990 und 2004 trugen die Defizite jedoch nur 65\% zum Schuldenanstieg bei. Die restlichen $35 \%$ erklären sich durch Sonderrechnungen außerhalb des ordentlichen Budgets, Darlehen, Beteiligungen und Veränderungen der kurzfristig verfügbaren Mittel (EFD 2006). Die positive und rasche Wirkung der automatischen Stabilisatoren wird durchwegs bestätigt, beim diskretionären Impuls erhalten wir in Gleichung (10) sogar ein negatives Vorzeichen. Steuererhöhungen im Jahr der Rezession wirken sich stets negativ aus, während die positive Wirkung der Geldpolitik nachgewiesen werden kann. Durch das Weglassen der Schuldenvariablen hat die Höhe der Staatsquote nun einen statistisch signifikanten negativen Einfluss auf das Wachstum pro Kopf.

\section{Schlussfolgerungen}

In diesem Paper haben wir die Stabilisierungswirkung der Schweizer Fiskalpolitik seit 1950 untersucht. Trotz verfassungsmäßigem Auftrag verfolgte der Bund nur in rund $60 \%$ der Fälle eine antizyklische Finanzpolitik. In Rezessionsjahren war diese jedoch immer antizyklisch. Seit 1990 und insbesondere seit der Einführung der Schuldenbremse im Jahr 2003 ist ein deutlicher Trend zur verstärkten Stabilisierung festzustellen. Ähnliches gilt für die Kantone, deren Fiskalpolitik über den Zeitraum von 1971 - 2005 in etwa gleich häufig antizyklisch wie prozyklisch und in Rezessionsjahren zu 54\% antizyklisch wirkte. Auch hier ist in den letzten Jahren ein Trend in Richtung antizyklischer Politik zu beobachten.

Die automatischen Stabilisatoren wurden insbesondere auf Bundesebene über die letzten 50 Jahre stark ausgebaut. Ihr Umfang ist rund doppelt so hoch wie die diskretionären Maßnahmen. Bezüglich makroökonomischer Wirkung schneiden die automatischen Stabilisatoren zudem besser ab. Sie wirken schnell, gezielt und befristet, während die diskretionäre Fiskalpolitik häufig verzögert eintritt und damit vor allem in kurzen und milden Rezessionen prozyklisch ist. Der Einfluss diskretionärer Maßnahmen auf die Konjunktur ist relativ

13. Dementsprechend sind die Gleichungen (9) und (10), (11) und (12) etc. direkt miteinander vergleichbar. 


\section{Fiskalpolitik als antizyklisches Instrument?}

bescheiden, die langfristigen Multiplikatoren liegen in allen Modellen unter eins. Es kann vermutet werden, dass dieser Effekt in den letzten Jahren aufgrund der zunehmenden internationalen Verflechtung und der damit verbundenen Offenheit der Wirtschaft weiter abgenommen hat. Zusammen mit der Geldpolitik sind die automatischen Stabilisatoren daher effektivere Konjunkturstützen. Diskretionäre fiskalpolitische Maßnahmen bergen außerdem das Risiko eines permanent höheren Ausgaben-, Steuer- und Schuldenniveaus in sich. Um ihrem verfassungsmäßigen Auftrag gerecht zu werden, ohne das Ziel der Nachhaltigkeit der öffentlichen Finanzen zu gefährden, sollten sich Bund und Kantone daher an den Kriterien orientieren, wonach entsprechende Maßnahmen zielgenau, zeitgerecht und zeitlich befristet sein müssen. Zu einer erfolgreichen antizyklischen Fiskalpolitik gehört letztendlich vor allem auch die Zurückhaltung bei weiteren Ausgaben in der Hochkonjunktur. Im Gegensatz zum Verhalten in Rezessionsphasen besteht hier sowohl beim Bund als auch bei den Kantonen weiterhin Handlungsbedarf.

\section{Literaturverzeichnis}

Ammann, Y. (2002), Quelques réflexions à propos des règles de politique budgétaire. Seco WP Discussion Paper No. 13, Bern. http://www.seco.admin.ch/dokumentation/ publikation/00011/00033/01763/index.html?lang=fr.

Arellano, M. und S. Bond (1991), Some Tests of Specification for Panel Data: Monte Carlo Evidence and an Application to Employment Equations, Review of Economic Studies 58, 277-297.

Balastèr, P. (2009), Die konjunkturpolitisch motivierte Finanzpolitik des Bundes seit 1975, Volkswirtschaft 82, 26-30.

Barro, R.J. (1974), Are Government Bonds Net Wealth?, Journal of Political Economy 82, 10951117.

Barro, R. J. (2009), Government Spending Is No Free Lunch, Wall Street Journal vom 22. Januar 2009, A.17.

Blanchard, O. (1990), Suggestions for a New Set of Fiscal Indicators. OECD Working Paper No. 79.

Blanchard, O. und R. Perotti (2002), An Empirical Characterization of the Dynamic Effects of Changes in Government Spending and Taxes on Output, Quarterly Journal of Economics 117, 1329-1368.

Blundell, R. und S. Bond (1998), Initial Conditions and Moment Restrictions in Dynamic Panel Data Models, Journal of Econometrics 87, 115-143.

Bruchez, P.-A. (2003), A Modification of the HP Filter Aiming at Reducing the End-Point Bias, Eidgenössische Finanzverwaltung, Working Paper, 18. August 2003

Bruchez, P.-A., C. Colombier, A. Geier, B. Schlaffer und A. Rey (2009), Politique conjoncturelle de la Confédération. EFV Working Paper No. 11.

Bundesamt für Sozialversicherungen [BSV] (2009), Schweizerische Sozialversicherungsstatistik 2008. BSV, Bern.

Bundesamt für Statistik [BFS] (2008), Landesindex der Konsumentenpreise. Indikatoren Indexreihen. http://www.bfs.admin.ch/bfs/portal/de/index/themen/05/02/blank/key/ basis_aktuell.html.

CESifo Forum 10/2 (2009), Fiscal Policy in Recession, München

Cwik, T. und Wieland, V. (2009), Keynesian Government Spending Multipliers and Spillovers in the Euro Area, CEPR Discussion Paper No. 7389, London.

de Castro, F und Hernández de Cos, P. (2007), The Economic Effects of Fiscal Policy: The Case of Spain, Journal of Macroeconomics 30, 1005-1028. 


\section{Christoph A. Schaltegger und Martin Weder}

Eidgenössisches Finanzdepartement [EFD] (2006), Bericht des Bundesrats über die Schuldenentwicklung der öffentlichen Haushalte. http://www.efd.admin.ch/ dokumentation/zahlen/00578/01051/index.html?lang?de.

Eidgenössische Finanzverwaltung [EFV] (verschiedene Jahrgänge), Öffentliche Finanzen in der Schweiz. Neuchâtel: Bundesamt für Statistik.

Eidgenössische Finanzverwaltung [EFV] (2004), Eine Neubewertung der Schuldenbremse. EFV, Bern.

Elmendorf, D.W. und J. Furman (2008), If, When, How: A Primer on Fiscal Stimulus. The Hamilton Project - Brookings Institution, Washington, D.C.

Fatás, A. und I. Mihov (2001), The Effects of Fiscal Policy on Consumption and Employment: Theory and Evidence. CEPR Discussion Paper No. 2760.

Feld, L.P. und G. Kirchgässner (2008), On the Effectiveness of Debt Brakes: The Swiss Experience, in: R. Neck und J.-E. Sturm (Hrsg.), Sustainability of Public Debt. Cambridge, USA, MIT Press.

Frey, R. (2007), Die Finanzpolitik des Bundes seit 1960. Bericht im Auftrag der Kommission für Konjunkturfragen. CREMA, Basel.

Frick, A. und D. Lampart (2004), Indikatoren zum konjunkturpolitischen Impuls der Finanzpolitik. KOF Konjunktur Analysen 7/8.

Frick, A., M. Graff und I. Hartig (2009), Diskretionäre Fiskalpolitik: Pro und Kontra, KOFSpezialanalysen 2009 - SA 1.

ILO (2009), World of Work Report 2009: The Global Jobs Crisis and Beyond. ILO, Geneva.

IWF (2008), Fiscal Policy as a Countercyclical Tool, in: IMF (Hrsg.), World Economic Outlook, October 2008. IMF, Washington D.C.

IWF (2010), A Policy-Driven, Multispeed Recovery, World Economic Outlook, January 2010. IMF, Washington, D.C.

Jaeger, F. und B. Schips (1980), Zur ökonomischen Analyse einer Staatsverschuldung, ergänzt durch eine beispielhafte Untersuchung der Verhältnisse in der Schweiz. Rüegger, Diessenhofen.

Jordan, T.R. (1994), Der Stand der Schweizerischen Fiskalpolitik: Eine Analyse anhand neuer Fiskalindikatoren, Schweizerische Zeitschrift für Volkswirtschaft und Statistik 130, 193-206.

Kirchgässner, G. und J. Wolters (2006), Einführung in die moderne Zeitreihenanalyse. Franz Vahlen, München.

Lampart, D. (2005), Die konjunkturelle Ausrichtung der Schweizer Finanzpolitik im internationalen Vergleich: Ex-ante- vs. Ex-post-Betrachtung. KOF Arbeitspapiere Nr. 109.

Mileva, E. (2007), Using Arellano-Bond Dynamic Panel GMM Estimators in Stata. Tutorial with examples using Stata 9.0. Working Paper Fordham University.

Musgrave, R.A. (1959), The Theory of Public Finance. McGraw-Hill, New York.

OECD (2002), Economic Surveys of Switzerland. OECD, Paris.

OECD (2009), Effectiveness and Scope of Fiscal Stimulus, in: OECD (Hrsg.), Economic Outlook, Interim Report. OECD, Paris.

Peacock, A.T. und J. Wiseman (1961), The Growth of Public Expenditure in the United Kingdom. Princeton University Press, Princeton.

Perotti, R. (2005), Estimating the Effects of Fiscal Policy in OECD Countries. CEPR Discussion Papers, No. 4842, London.

Ramey, V.A. und M.D. Shapiro (1998), Costly Capital Reallocation and the Effects of Government Spending, Carnegie-Rochester Conference Series on Public Policy 48, 145-194.

Reinhart, C. und K. Rogoff (2009), The Aftermath of Financial Crises. NBER Working Paper No. 14656.

Roodman, D. (2006), How to Do xtabond2: An Introduction to "Difference" and "System" GMM in Stata. Center for Global Development Working Paper Number 103.

Schweizerische Nationalbank [SNB] (2007), Die Schweizerische Nationalbank 1907-2007. NZZ Libro, Schlieren.

Schweizerische Nationalbank [SNB] (2009), Statistische Publikationen: Historische Zeitreihen. Gefunden am 27. Januar 2009 unter http://www.snb.ch/de/iabout/stat/ statpub/histz/id/statpub_histz_actual 


\title{
Fiskalpolitik als antizyklisches Instrument?
}

Shapiro, M. D. und J. B. Slemrod (2002), Did the 2001 Tax Rebate Stimulate Spending? Evidence from Taxpayer Surveys, NBER Working Paper No. 9308, Cambridge, MA.

Staatssekretariat für Wirtschaft [Seco] (2009), BIP und Verwendungskomponenten (Jahres- und Quartalsdaten). Gefunden am 14. April 2009 unter http://www. seco.admin.ch/themen/00374/00456/00458/index.html?lang=de

Stone, C. und K. Cox (2008), Economic Policy in a Weakening Economy: Principles of Fiscal Stimulus. Center on Budget and Policy Priorities, Washington, D.C.

\begin{abstract}
This paper examines the stabilizing effects of Swiss fiscal policy. First, we find that the federation adopted a countercyclical fiscal policy in approximately $60 \%$ of all periods observed. During recessions, fiscal policy was always countercyclical and therefore helped to stabilize the economy. In case of the cantons, fiscal policy was countercyclical in 55\% of all recessions. In recent years, there has been a trend for both the federation and the cantons toward stronger stabilization. Second, the two stabilizing instruments of fiscal policy, automatic stabilizers and discretionary fiscal policy are compared with each other. Over the last 50 years, automatic stabilizers have been expanded continuously, particularly on the federal level. We find that the impulse of automatic stabilizers is about twice as large as the one of discretionary fiscal policy. Third, macroeconomic effects of Switzerland's fiscal policy during recessions are examined. Automatic stabilizers have been particularly effective in the cantons whereas the effects of discretionary fiscal policy on economic growth have tended to be weaker.
\end{abstract}

\section{Anhang A: Datenbeschreibung}

\begin{tabular}{lcc}
\hline Variable & \multicolumn{1}{c}{ Beschreibung } & Quellen \\
\hline Wachstum & Wachstum des realen BIP bzw. des & Seco, Bundesamt für \\
& realen kantonalen & Statistik, eigene \\
& Volkseinkommens & Berechnungen \\
Pro-Kopf-Wachstum & Wachstum des realen BIP pro Kopf & Seco, Bundesamt für \\
& bzw. reales Volkseinkommen pro & Statistik, eigene \\
& Kopf & Berechnungen \\
Einnahmen & Veränderung der realen Einnahmen & EFV, Bundesamt für \\
& in Prozentpunkten des BIP bzw. & Statistik, eigene \\
& des kantonalen Volkseinkommens & Berechnungen \\
Vusgaben & Veränderung der realen & EFV, Bundesamt für \\
& Primärausgaben in & Statistik, eigene \\
& Prozentpunkten des BIP bzw. des & Berechnungen \\
kantonalen Volkseinkommens & \\
Impuls & Nettoeffekt diskretionärer & EFV, Bundesamt für \\
& Veränderungen auf der & Statistik, eigene \\
& Einnahmen und Ausgabenseite & Berechnungen \\
& während einer Rezession in & \\
& Prozentpunkten des BIP bzw. des & \\
& kantonalen Volkseinkommens & \\
& Nettoeffekt der automatischen & EFV, Bundesamt für \\
Stabilisatoren während einer & Statistik, eigene \\
& Rezession in Prozentpunkten des & Berechnungen \\
& BIP bzw. des kantonalen & \\
Volkseinkommens & \\
\hline
\end{tabular}


Anhang A: (Continued)

\begin{tabular}{|c|c|c|}
\hline Variable & Beschreibung & Quellen \\
\hline Bevölkerungswachstum & $\begin{array}{l}\text { Wachstumsrate der ständigen } \\
\text { Wohnbevölkerung in Prozent } \\
\text { gegenüber dem Vorjahr }\end{array}$ & $\begin{array}{l}\text { Bundesamt für Statistik, } \\
\text { eigene Berechnungen }\end{array}$ \\
\hline Geldpolitik & $\begin{array}{l}\text { Veränderung des Leitzinssatzes der } \\
\text { Schweizerischen Nationalbank } \\
\text { (SNB) gegenüber dem Vorjahr }\end{array}$ & SNB \\
\hline Investitionen & $\begin{array}{l}\text { Veränderungen der Investitionen } \\
\text { gegenüber dem Vorjahr in } \\
\text { Prozentpunkten des BIP }\end{array}$ & $\begin{array}{r}\text { Penn World Tables } 6.2 \text {, } \\
\text { eigene Berechnungen }\end{array}$ \\
\hline Weltwirtschaft & $\begin{array}{l}\text { Reale Wachstumsrate des } \\
\text { Bruttoinlandproduktes der Welt }\end{array}$ & IWF \\
\hline Staatsquote & $\begin{array}{l}\text { Reale Gesamtausgaben in Prozent } \\
\text { des kantonalen Volkseinkommens }\end{array}$ & $\begin{array}{l}\text { EFV, Bundesamt für } \\
\text { Statistik, eigene } \\
\text { Berechnungen }\end{array}$ \\
\hline Volkseinkommen & $\begin{array}{l}\text { Logarithmiertes reales kantonales } \\
\text { Volkseinkommen }\end{array}$ & $\begin{array}{l}\text { Bundesamt für Statistik, } \\
\text { eigene Berechnungen }\end{array}$ \\
\hline Schulden & $\begin{array}{l}\text { Veränderung der Schulden } \\
\text { gegenüber dem Vorjahr in } \\
\text { Prozentpunkten des BIP bzw. des } \\
\text { kantonalen Volkseinkommens }\end{array}$ & $\begin{array}{l}\text { EFV, Bundesamt für } \\
\text { Statistik, eigene } \\
\text { Berechnungen }\end{array}$ \\
\hline
\end{tabular}

Anhang B: Deskriptive Statistik

\begin{tabular}{|c|c|c|c|c|}
\hline Variable & Mittelwert & Std, $A b w$, & Minimum & Maximum \\
\hline \multicolumn{5}{|l|}{ Bund: } \\
\hline Wachstum pro Kopf & 0,018 & 0,023 & $-0,062$ & 0,062 \\
\hline Einnahmen & 0,000 & 0,007 & $-0,018$ & 0,018 \\
\hline Ausgaben & 0,000 & 0,005 & $-0,014$ & 0,014 \\
\hline Impuls & 0,000 & 0,001 & $-0,007$ & 0,004 \\
\hline Stabilisatoren & $-0,001$ & 0,003 & $-0,013$ & 0,006 \\
\hline Bevölkerung & 0,008 & 0,006 & $-0,006$ & 0,028 \\
\hline Geldpolitik & 0,022 & 0,997 & $-2,500$ & 3,000 \\
\hline Investitionen & 0,128 & 1,966 & $-6,430$ & 4,110 \\
\hline Weltwirtschaft & 0,040 & 0,013 & 0,009 & 0,073 \\
\hline Schulden & $-0,003$ & 0,016 & $-0,035$ & 0,036 \\
\hline \multicolumn{5}{|l|}{ Kantone: } \\
\hline Wachstum & 0,011 & 0,039 & $-0,167$ & 0,410 \\
\hline Einnahmen & 0,002 & 0,014 & $-0,095$ & 0,092 \\
\hline Ausgaben & 0,002 & 0,013 & $-0,099$ & 0,088 \\
\hline Impuls & 0,000 & 0,009 & $-0,061$ & 0,090 \\
\hline Stabilisatoren & $-0,000$ & 0,009 & $-0,088$ & 0,055 \\
\hline Bevölkerung & 0,006 & 0,017 & $-0,229$ & 0,175 \\
\hline Geldpolitik & $-0,079$ & 1,203 & $-2,500$ & 3,000 \\
\hline Staatsquote & 0,171 & 0,062 & 0,062 & 0,572 \\
\hline Volkseinkommen & 15,637 & 1,185 & 12,695 & 18,182 \\
\hline Schulden & $-0,001$ & 0,021 & $-0,166$ & 0,241 \\
\hline
\end{tabular}


Fiskalpolitik als antizyklisches Instrument?

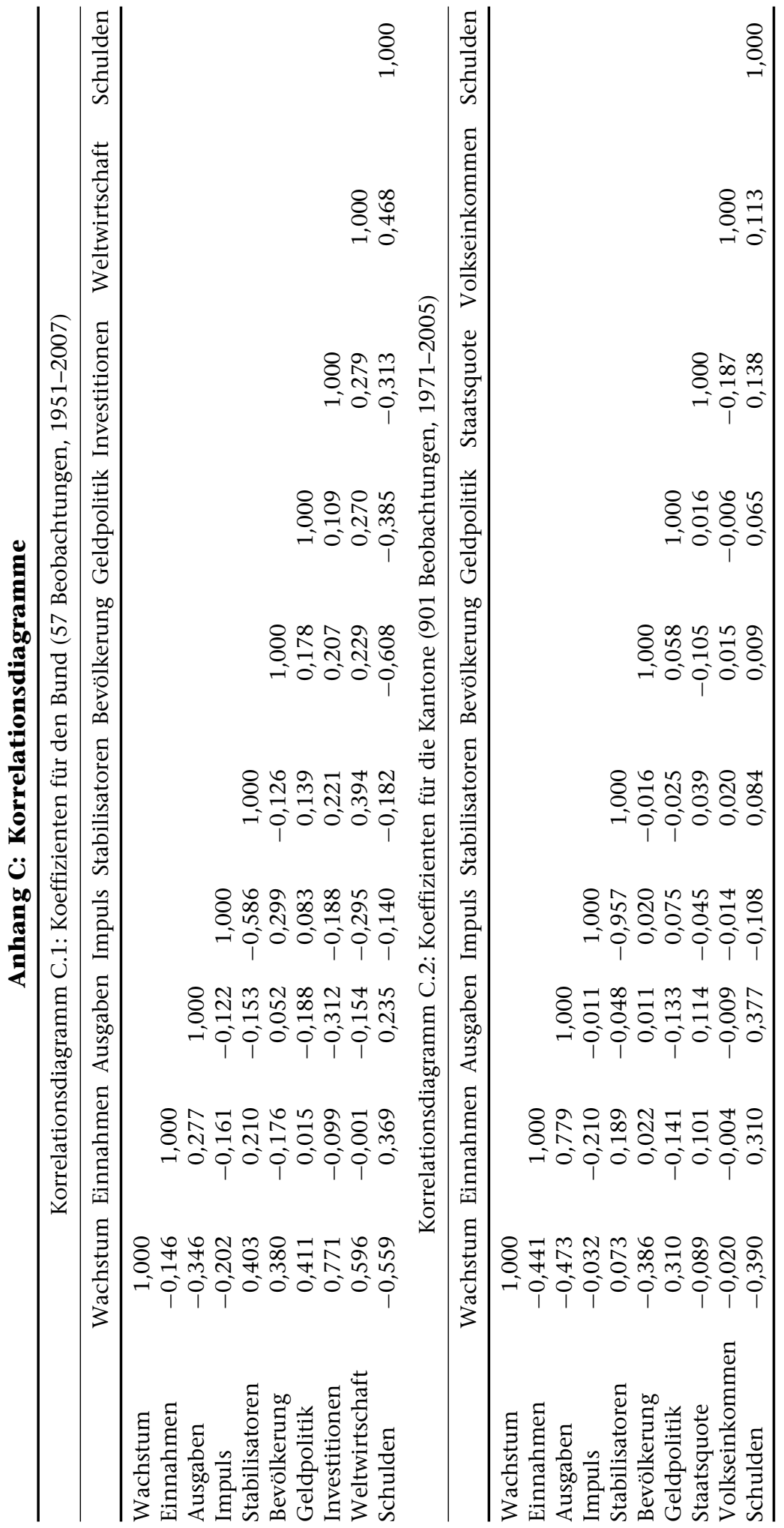




\section{Christoph A. Schaltegger und Martin Weder}

Anhang D: Tests zur Überprüfung der Stationarität

\begin{tabular}{|c|c|c|c|c|}
\hline \multirow{2}{*}{$\begin{array}{l}\text { Variablen } \\
\text { (1951-2007) }\end{array}$} & \multicolumn{4}{|c|}{ Augmented Dickey-Fuller Test (2 Lags) } \\
\hline & Level & Level mit Trend & Differenzen & Diff, Trend \\
\hline $\begin{array}{l}\text { BIP pro Kopf } \\
\text { Primärausgaben } \\
\text { Einnahmen }\end{array}$ & $\begin{array}{l}-1,384 \\
-1,213 \\
-0,604\end{array}$ & $\begin{array}{l}-2,215 \\
-2,307 \\
-2,769\end{array}$ & $\begin{array}{l}-3,725^{* * *} \\
-4,206^{* * *} \\
-4,661^{* * *}\end{array}$ & $\begin{array}{l}-4,529^{* * *} \\
-4,141^{* * *} \\
-4,704^{* * *}\end{array}$ \\
\hline \multirow{2}{*}{$\begin{array}{l}\text { Variablen } \\
\text { (1951-1972) }\end{array}$} & \multicolumn{4}{|c|}{ Augmented Dickey-Fuller Test (2 Lags) } \\
\hline & Level & Level mit Trend & Differenzen & Diff, Trend \\
\hline $\begin{array}{l}\text { BIP pro Kopf } \\
\text { Primärausgaben } \\
\text { Einnahmen }\end{array}$ & $\begin{aligned} & 1,127 \\
&-0,560 \\
&-3,897^{* * *}\end{aligned}$ & $\begin{array}{l}-1,592 \\
-3,223 \\
-3,973^{* *}\end{array}$ & $\begin{array}{l}-4,224^{* * *} \\
-2,820^{*} \\
-5,343^{* * *}\end{array}$ & $\begin{array}{l}-4,080^{* *} \\
-2,893 \\
-5,424^{* * *}\end{array}$ \\
\hline \multirow{2}{*}{$\begin{array}{l}\text { Variablen } \\
(1973-2007)\end{array}$} & \multicolumn{4}{|c|}{ Augmented Dickey-Fuller Test (2 Lags) } \\
\hline & Level & Level mit Trend & Differenzen & Diff, Trend \\
\hline $\begin{array}{l}\text { BIP pro Kopf } \\
\text { Primärausgaben } \\
\text { Einnahmen }\end{array}$ & $\begin{array}{r}0,316 \\
-1,769 \\
-1,195\end{array}$ & $\begin{array}{l}-2,838 \\
-1,654 \\
-2,612\end{array}$ & $\begin{array}{l}-3,843^{* * *} \\
-2,818^{*} \\
-3,253^{* *}\end{array}$ & $\begin{array}{l}-3,729^{* *} \\
-2,960 \\
-3,190\end{array}$ \\
\hline \multirow{2}{*}{$\begin{array}{l}\text { Variablen } \\
(1951-2007)\end{array}$} & \multicolumn{4}{|c|}{ Phillips-Peron Test (2 Lags) } \\
\hline & Level & Level mit Trend & Differenzen & Diff, Trend \\
\hline $\begin{array}{l}\text { BIP pro Kopf } \\
\text { Primärausgaben } \\
\text { Einnahmen }\end{array}$ & $\begin{array}{l}-1,049 \\
-1,302 \\
-1,275\end{array}$ & $\begin{array}{l}-1,980 \\
-3,046 \\
-4,613^{* * *}\end{array}$ & $\begin{array}{r}-5,374^{* * *} \\
-9,108^{* * *} \\
-17,043^{* * *}\end{array}$ & $\begin{array}{r}-5,634^{* * *} \\
-9,042^{* * *} \\
-17,255^{* * *}\end{array}$ \\
\hline \multirow{2}{*}{$\begin{array}{l}\text { Variablen } \\
(1951-1972)\end{array}$} & \multicolumn{4}{|c|}{ Phillips-Peron Test (2 Lags) } \\
\hline & Level & Level mit Trend & Differenzen & Diff, Trend \\
\hline $\begin{array}{l}\text { BIP pro Kopf } \\
\text { Primärausgaben } \\
\text { Einnahmen }\end{array}$ & $\begin{array}{c}1,564 \\
-1,450 \\
-10,071^{* * *}\end{array}$ & $\begin{array}{c}-2,060 \\
-2,675 \\
-10,313^{* * *}\end{array}$ & $\begin{array}{r}-4,308^{* * *} \\
-6,351^{* * *} \\
-20,241^{* * *}\end{array}$ & $\begin{array}{c}-4,256^{* *} \\
-6,406^{* * *} \\
-20,269^{* * *}\end{array}$ \\
\hline \multirow{2}{*}{$\begin{array}{l}\text { Variablen } \\
(1973-2007)\end{array}$} & \multicolumn{4}{|c|}{ Phillips-Peron Test (2 Lags) } \\
\hline & Level & Level mit Trend & Differenzen & Diff, Trend \\
\hline \multirow{3}{*}{$\begin{array}{l}\text { BIP pro Kopf } \\
\text { Primärausgaben } \\
\text { Einnahmen } \\
\text { Variablen (1951-2007) } \\
\end{array}$} & $\begin{array}{r}0,327 \\
-1,876 \\
-1,419\end{array}$ & $\begin{array}{l}-2,424 \\
-1,898 \\
-3,713^{* *}\end{array}$ & $\begin{array}{r}-4,277^{* * *} \\
-6,141^{* * *} \\
-10,922^{* * *}\end{array}$ & $\begin{array}{c}-4,254^{* *} \\
-6,252^{* * *} \\
-10,767^{* * *}\end{array}$ \\
\hline & & \multicolumn{3}{|c|}{$\begin{array}{l}\text { Kwiatkowski-Philipps-Schmidt-Shin } \\
\text { (KPSS) Test (2 Lags) }\end{array}$} \\
\hline & & Level & & Differenzen \\
\hline $\begin{array}{l}\text { BIP pro Kopf } \\
\text { Primärausgaben } \\
\text { Einnahmen }\end{array}$ & & $\begin{array}{l}0,365^{* * *} \\
0,079 \\
0,249^{* * *}\end{array}$ & & $\begin{array}{l}0,071 \\
0,070 \\
0,043\end{array}$ \\
\hline
\end{tabular}

KPSS-Test: Nullhypothese, dass Variable trendstationär ist

Kritische Werte für 1\%: 0,216, 5\%: 0,146, 10\%: 0,119 
Fiskalpolitik als antizyklisches Instrument?

Anhang D: (Continued)

\begin{tabular}{llc}
\hline & \multicolumn{2}{c}{$\begin{array}{c}\text { Kwiatkowski-Philipps-Schmidt-Shin } \\
\text { (KPSS) Test (2 Lags) }\end{array}$} \\
\cline { 2 - 3 } Variablen (1951-1972) & Level & Differenzen \\
\hline BIP pro Kopf & $0,137^{*}$ & 0,048 \\
Primärausgaben & $0,129^{*}$ & 0,071 \\
Einnahmen & 0,086 & 0,065 \\
\hline
\end{tabular}

KPSS-Test: Nullhypothese, dass Variable trendstationär ist

Kritische Werte für 1\%: 0,216, 5\%: 0,146, 10\%: 0,119

Kwiatkowski-Philipps-Schmidt-Shin (KPSS) Test (2 Lags)

Variablen (1973-2007)

Level

Differenzen

BIP pro Kopf

0,069

0,058

Primärausgaben

0,119

0,100

Einnahmen

0,113

0,046

KPSS-Test: Nullhypothese, dass Variable trendstationär ist

Kritische Werte für 1\%: 0,216, 5\%: 0,146, 10\%: 0,119

\section{Anhang E: Grafische Darstellung des Clemente-Montañes-Reyes Test in Stata}

Clemente-Montañés-Reyes single AO test for unit root in series: gdp_cap_growth
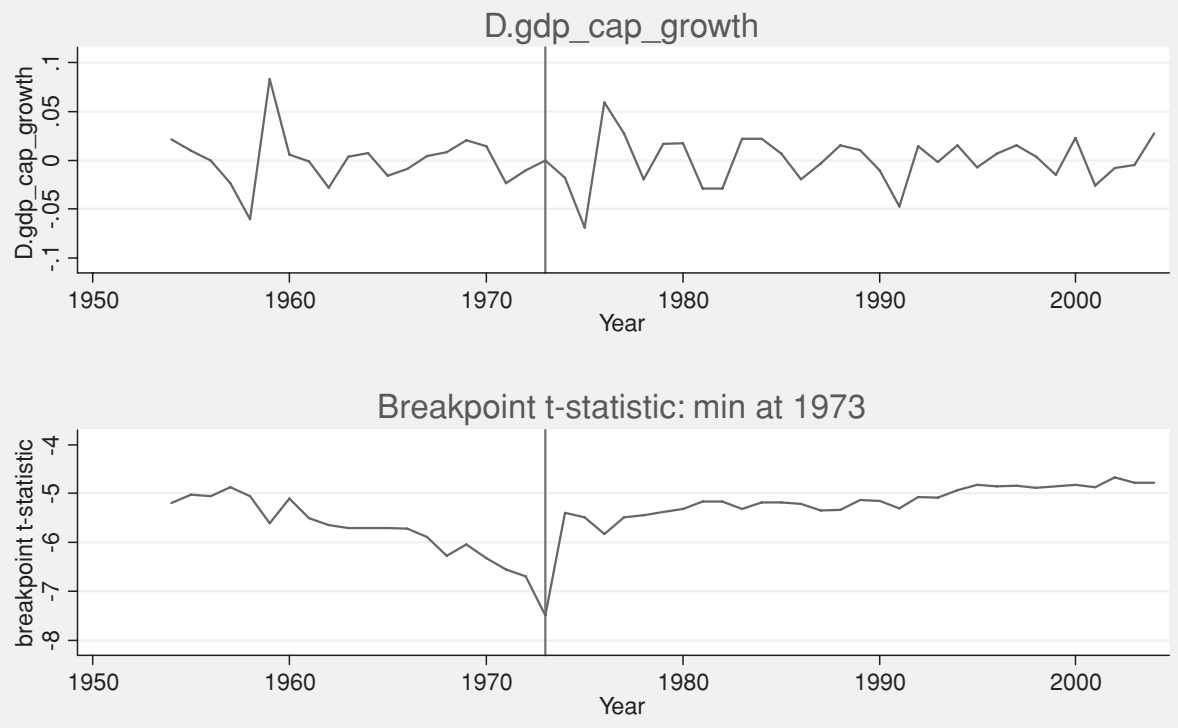